\title{
ADDITIVE REPRESENTATION IN THIN SEQUENCES, I: WARING'S PROBLEM FOR CUBES 해
}

\author{
BY JÖRG BRÜDERN，KOICHI KAWADA AND TREVOR D. WOOLEY
}

ABSTRACT. - In this paper we investigate representation of numbers from certain thin sequences like the squares or cubes by sums of cubes. It is shown, in particular, that almost all values of an integral cubic polynomial are sums of six cubes. The methods are very flexible and may be applied to many related problems.

(C) 2001 Éditions scientifiques et médicales Elsevier SAS

RÉSUMÉ. - Dans cet article nous étudions la représentation des nombres de certaines suites rares comme celles des carrés ou des cubes. Il est démontré notamment que presque toutes les valeurs d'un polynôme de degré trois sont des sommes de six cubes. Ces méthodes, très flexibles, sont applicables à beaucoup de problèmes analogues.

(c) 2001 Éditions scientifiques et médicales Elsevier SAS

\section{Introduction}

Throughout the history of the additive theory of numbers, the classical version of Waring's problem, and most notably problems involving sums of cubes, have provided the examples of prime importance for assessing the strength of novel methods. We will follow this tradition in the present communication. While our main objective is to present a widely applicable technique, we illustrate the latter solely in the context of sums of cubes, deferring to future memoirs discussion of the cornucopia of applications stemming from the underlying ideas. In advance of a detailed description of these ideas, it is appropriate first to review the state of the art in the class of examples we have in mind.

Let $R_{s}(n)$ denote the number of representations of $n$ as the sum of $s$ cubes of positive integers. It is widely believed that all large integers are represented as the sum of four cubes of positive integers, and indeed a heuristic application of the circle method motivates the conjecture that for $s \geqslant 4$, one has the asymptotic formula

$$
R_{s}(n)=\frac{\Gamma\left(\frac{4}{3}\right)^{s}}{\Gamma\left(\frac{s}{3}\right)} \mathfrak{S}_{s}(n) n^{\frac{s}{3}-1}+\mathrm{o}\left(n^{\frac{s}{3}-1}\right)
$$

\footnotetext{
The third author is a Packard Fellow, and is supported in part by NSF grant DMS-9622773. This paper benefitted from visits of various of the authors to Ann Arbor, Kyoto, Oberwolfach and Stuttgart, and the authors collectively thank these institutions for their hospitality and excellent working conditions.
} 
where

$$
\mathfrak{S}_{s}(n)=\sum_{q=1}^{\infty} \sum_{\substack{a=1 \\(a, q)=1}}^{q}\left(\frac{1}{q} \sum_{r=1}^{q} e\left(a r^{3} / q\right)\right)^{s} e(-a n / q),
$$

and $e(z)=\exp (2 \pi \mathrm{i} z)$. It is known that the singular series $\mathfrak{S}_{s}(n)$ satisfies $\mathfrak{S}_{s}(n) \gg 1$ for $s \geqslant 4$ (see Theorem 4.5 of Vaughan [17]). Consequently, the validity of formula (1.1) would imply that all large natural numbers are indeed the sum of four positive integral cubes.

The formula (1.1) has been established for $s \geqslant 9$ by Hardy and Littlewood [11], and by Vaughan [14] in the additional case $s=8$. Although no such asymptotic formula is known for $s \leqslant 7$, it does at least follow from work of Vaughan [16] that $R_{7}(n) \gg n^{4 / 3}$, which is the order of magnitude predicted by (1.1) (this conclusion improves on earlier work of Linnik [13], Watson [19], and Vaughan [14]). In situations where fewer cubes are employed in the representation, one must be content with rather weaker results. When $s$ is a natural number, denote by $E_{s}(N)$ the number of natural numbers not exceeding $N$ which are not the sum of $s$ cubes of positive integers. Then Davenport [8], in the first noteworthy contribution concerning sums of four cubes, established that $E_{4}(N) \ll N^{29 / 30+\varepsilon}$, showing in particular that almost all natural numbers are the sum of four positive integral cubes. The exponent 29/30 has subsequently been reduced, and it is now known that when $\varepsilon$ is a sufficiently small positive number, then one has

$$
E_{4}(N) \ll N^{37 / 42-\varepsilon}
$$

(this is a consequence of work of Brüdern [2] and Wooley [22]). The work of Vaughan [14] alluded to above, moreover, shows that formula (1.1) holds when $s=4$, for almost all $n$. The potential for more refined results along the lines of Davenport's theorem has recently received further attention, with work of Brüdern and Watt [7] and Kawada [12] concerning sums of four cubes in short intervals.

Although for sums of five or six cubes of non-negative integers results analogous to Davenport's theorem may be established through similar methods, one would anticipate that still stronger conclusions should be accessible given the additional variables available. This area remains, however, undeservedly neglected in the literature. In this context we remark that by combining use of Weyl's inequality for the additional cubic exponential sums with the classical approach leading to the estimate (1.2), one routinely obtains bounds very slightly sharper than

$$
E_{5}(N) \ll N^{5 / 7}, \quad E_{6}(N) \ll N^{23 / 42} .
$$

As is evident from (1.3), these classical estimates fail to show even that the majority of integer squares are represented as the sum of six positive cubes. Nevertheless, as a consequence of the methods developed within this paper, it is possible to show that almost all values of a quadratic polynomial are the sum of six cubes, with an explicit estimate for the size of the exceptional set. In order to state our result in a precise form, it is convenient henceforth to describe a polynomial $\phi \in \mathbb{Q}[t]$ as being an integral polynomial if, whenever the parameter $t$ is an integer, then the value $\phi(t)$ is also an integer. When $\phi(t)$ is such a polynomial, denote by $E_{\phi}(N)$ the number of integers $n$ with $1 \leqslant n \leqslant N$ for which $\phi(n)$ is not the sum of six cubes of positive integers.

THEOREM 1.1. - Let $\phi$ be an integral quadratic polynomial with positive leading coefficient. Then one has $E_{\phi}(N) \ll_{\phi} N^{19 / 28}$.

When the quadratic polynomial is replaced by a cubic polynomial, it is still possible to obtain a similar conclusion, but with a weaker estimate for the number of exceptions. 
THEOREM 1.2. - Let $\Phi$ be an integral cubic polynomial with positive leading coefficient. Then one has $E_{\Phi}(N) \ll_{\Phi} N^{255 / 274}$.

A trivial variant of the arguments used to establish Theorem 1.2 shows that when $N$ is a positive integer, then for almost all integers $t$ with $1 \leqslant t \leqslant N^{1 / 3}$, one has that $N-t^{3}$ is the sum of six positive integral cubes. Thus we have the curious conclusion that not only does one have that $R_{7}(N)>0$ for all large integers $N$, but also that one can almost prescribe the value of one of the seven cubes used to represent $N$.

Our methods offer great flexibility in their application to the study of exceptional sets in additive number theory. In principle, the proofs of the two theorems above might be regarded as providing a model for most applications of our method. It therefore seems appropriate to indulge in some rather abstract discussion concerning our methods, and in particular to describe their merits relative to classical approaches involving Bessel's inequality.

We are interested in additive representations of integers. Thus, given a positive integer $n$ and sets $\mathcal{A}_{1}, \ldots, \mathcal{A}_{s}$ of positive integers, we investigate the number $r(n)$ of representations of $n$ in the shape

$$
n=a_{1}+a_{2}+\cdots+a_{s}
$$

with $a_{i} \in \mathcal{A}_{i}(1 \leqslant i \leqslant s)$. When $\mathcal{B} \subseteq \mathbb{N}$ we define $E_{\mathcal{B}}(X)$ to be the number of integers $n \in[1, X] \cap \mathcal{B}$ having no representation in the shape (1.4). There are many circumstances in which for some fixed $s$, and an interesting choice of $\mathcal{A}_{1}, \ldots, \mathcal{A}_{s}$, one is able to show that almost all positive integers $n$ possess a representation in the form (1.4), whence

$$
E_{\mathbb{N}}(X)=\mathrm{o}(X)
$$

If, on the other hand, one is able to show that for some thin set $\mathcal{B}$, such as the sequence of squares, one has

$$
E_{\mathcal{B}}(X)=\mathrm{o}(\operatorname{card}(\mathcal{B} \cap[1, X])),
$$

then one can reasonably assert that more refined information is being provided concerning the distribution of integers represented in the form (1.4). Such problems form the backbone of this series of papers.

It is informative to describe the classical approach to estimating $E_{\mathbb{N}}(X)$ in order that its limitations be evident. Define the generating functions

$$
f_{i}(\alpha)=\sum_{x \in \mathcal{A}_{i} \cap[1, X]} e(\alpha x) \quad(1 \leqslant i \leqslant s) .
$$

Also, define the mean value $r_{\mathfrak{C}}(n)$ relative to a measurable set $\mathfrak{C} \subseteq[0,1]$ by

$$
r_{\mathfrak{C}}(n)=\int_{\mathfrak{C}} f_{1}(\alpha) \cdots f_{\mathcal{s}}(\alpha) e(-\alpha n) \mathrm{d} \alpha .
$$

Then by orthogonality one has $r(n)=r_{[0,1]}(n)$. The first idea in the classical approach is to use the Hardy-Littlewood method to estimate $r(n)$. Thus one divides the unit interval into complementary sets $\mathfrak{M}$ and $\mathfrak{m}$, on the first of which one hopes to provide asymptotic formulae for the $f_{i}(\alpha)$, and on the second we aim to show that the generating functions are on average suitably small. Under favourable conditions one is able to show that for $\frac{1}{2} X<n \leqslant X$ one has 
$r_{\mathfrak{M}}(n) \asymp \Upsilon(X)$, where

$$
\Upsilon(X)=X^{-1} \prod_{i=1}^{s} \operatorname{card}\left(\mathcal{A}_{i} \cap[1, X]\right) .
$$

Meanwhile, an application of Bessel's inequality reveals that

$$
\sum_{n \in \mathbb{Z}}\left|r_{\mathfrak{m}}(n)\right|^{2} \leqslant \int_{\mathfrak{m}}\left|f_{1}(\alpha) \cdots f_{s}(\alpha)\right|^{2} \mathrm{~d} \alpha
$$

Once again under suitable conditions, one is able to show that the latter expression is of order strictly smaller than $X \Upsilon(X)^{2}$. On recalling (1.6) and (1.7), therefore, we find that

$$
\sum_{\frac{1}{2} X<n \leqslant X}\left|\frac{r(n)-r_{\mathfrak{M}}(n)}{r_{\mathfrak{M}}(n)}\right|^{2} \ll \Upsilon(X)^{-2} \sum_{n}\left|r_{\mathfrak{m}}(n)\right|^{2}=\mathrm{o}(X) .
$$

On noting that the summands on the left hand side of (1.8) are equal to 1 whenever $r(n)=0$, and otherwise are non-negative, we conclude that the right hand side of (1.8) provides an upper bound for $E_{\mathbb{N}}(X)-E_{\mathbb{N}}\left(\frac{1}{2} X\right)$. By summing over dyadic intervals, it is therefore evident how, in principle, one may establish a result of the type (1.5).

Although successful in investigations concerning exceptional sets of the type $E_{\mathbb{N}}(X)$, the above method fails to provide strong conclusions for exceptional sets $E_{\mathcal{B}}(X)$ when $\mathcal{B}$ is a thin sequence. The difficulty is that Bessel's inequality fails to exploit the size of the set $\mathcal{B}$, or indeed its arithmetic properties, and thus the "trivial" estimate arising from the above method may be far larger than the obvious upper bound $\operatorname{card}(\mathcal{B} \cap[1, X])$ for $E_{\mathcal{B}}(X)$.

In this paper we investigate the "sequence of exceptions" directly. Define $\mathcal{E}$ to be the set of integers $n \in \mathcal{B}$ having no representation in the shape (1.4). Then by orthogonality it follows from (1.4) that

$$
\sum_{\substack{n \in \mathcal{E} \\ \frac{1}{2} X<n \leqslant X}} \int_{0}^{1} f_{1}(\alpha) \cdots f_{s}(\alpha) e(-\alpha n) \mathrm{d} \alpha=0
$$

Write

$$
K(\alpha)=\sum_{\substack{n \in \mathcal{E} \\ \frac{1}{2} X<n \leqslant X}} e(\alpha n),
$$

and note that under the conditions implicit in the classical argument above, one has

$$
\sum_{\substack{n \in \mathcal{E} \\ \frac{1}{2} X<n \leqslant X}} \int_{\mathfrak{M}} f_{1}(\alpha) \cdots f_{s}(\alpha) e(-\alpha n) \mathrm{d} \alpha \gg \Upsilon(X) \operatorname{card}\left(\mathcal{E} \cap\left(\frac{1}{2} X, X\right]\right) .
$$

But from (1.9) and (1.10) it follows that

$$
\sum_{\substack{n \in \mathcal{E} \\ \frac{1}{2} X<n \leqslant X}} \int_{\mathfrak{M}} f_{1}(\alpha) \cdots f_{s}(\alpha) e(-\alpha n) \mathrm{d} \alpha=-\int_{\mathfrak{m}} f_{1}(\alpha) \cdots f_{s}(\alpha) K(-\alpha) \mathrm{d} \alpha,
$$


whence by (1.11),

$$
E_{\mathcal{B}}(X)-E_{\mathcal{B}}\left(\frac{1}{2} X\right) \ll \Upsilon(X)^{-1} \int_{\mathfrak{m}}\left|f_{1}(\alpha) \cdots f_{s}(\alpha) K(\alpha)\right| \mathrm{d} \alpha .
$$

In this way, the conclusion of the classical treatment may be recovered by applying Schwarz's inequality to the integral on the right hand side of (1.12), since by orthogonality,

$$
\begin{aligned}
& \int_{\mathfrak{m}}\left|f_{1}(\alpha) \cdots f_{s}(\alpha) K(\alpha)\right| \mathrm{d} \alpha \\
& \leqslant\left(\int_{0}^{1}|K(\alpha)|^{2} \mathrm{~d} \alpha\right)^{1 / 2}\left(\int_{\mathfrak{m}}\left|f_{1}(\alpha) \cdots f_{s}(\alpha)\right|^{2} \mathrm{~d} \alpha\right)^{1 / 2} \\
& \leqslant\left(E_{\mathcal{B}}(X)-E_{\mathcal{B}}\left(\frac{1}{2} X\right)\right)^{1 / 2}\left(\int_{\mathfrak{m}}\left|f_{1}(\alpha) \cdots f_{s}(\alpha)\right|^{2} \mathrm{~d} \alpha\right)^{1 / 2} .
\end{aligned}
$$

However, the formulation (1.12) offers additional flexibility over the classical treatment whenever the set $\mathcal{B}$ possesses useful arithmetic properties. For example, if $\mathcal{B}$ is the set of integral squares, then the fourth moment of $K(\alpha)$ may be estimated non-trivially, and then the upper bound (1.12) may become effective via an application of Hölder's inequality. This is essentially the strategy adopted in the proof of Theorem 1.1. To be specific in the case of squares under consideration, a straightforward counting argument shows that

$$
\int_{0}^{1}|K(\alpha)|^{4} \mathrm{~d} \alpha \ll X^{\varepsilon}\left(E_{\mathcal{B}}(X)-E_{\mathcal{B}}\left(\frac{1}{2} X\right)\right)^{2}
$$

and (1.12) then yields, via Hölder's inequality,

$$
E_{\mathcal{B}}(X)-E_{\mathcal{B}}\left(\frac{1}{2} X\right) \ll X^{\varepsilon} \Upsilon(X)^{-2}\left(\int_{\mathfrak{m}}\left|f_{1}(\alpha) \cdots f_{s}(\alpha)\right|^{4 / 3} \mathrm{~d} \alpha\right)^{3 / 2} .
$$

There are numerous alternatives to the latter approach which vary in utility according to the mean value estimates available. It may be useful to consider mixed mean values of the shape

$$
\int_{0}^{1}\left|K(\alpha) f_{i_{1}}(\alpha) \cdots f_{i_{t}}(\alpha)\right|^{2} \mathrm{~d} \alpha
$$

wherein the exponential sum $K(\alpha)$ may, if desired, be replaced by the complete sum

$$
\sum_{\substack{n \leqslant X \\ n \in \mathcal{B}}} e(\alpha n)
$$

by considering the underlying diophantine equation. A very successful application of this mixed mean value idea is to problems involving mixed powers, as will be demonstrated in a sequel to 
this paper [6]. Moreover, it is possible to incorporate efficient differencing into this approach, as will be evident from our proof of Theorem 1.2.

There is an alternative, more classical approach to these problems which we mention before moving on, and this approach is the one exploited by earlier writers. One can make use of relation (1.8), but now expanding the central expression in (1.8) to obtain for a general sequence $\mathcal{B}$ in place of $\mathbb{N}$,

$$
\sum_{\substack{n \in \mathcal{B} \\ \frac{1}{2} X<n \leqslant X}}\left|\frac{r(n)-r_{\mathfrak{M}}(n)}{r_{\mathfrak{M}}(n)}\right|^{2} \ll \Upsilon(X)^{-2} I(X)
$$

where

$$
I(X)=\int_{\mathfrak{m}} \int_{\mathfrak{m}} f_{1}(\alpha) f_{1}(-\beta) \cdots f_{s}(\alpha) f_{s}(-\beta) \sum_{\substack{n \in \mathcal{B} \\ \frac{1}{2} X<n \leqslant X}} e((\beta-\alpha) n) \mathrm{d} \alpha \mathrm{d} \beta .
$$

As an advantage over our approach sketched above, an exponential sum over all elements of $\mathcal{B}$ in an interval occurs (a classical Weyl sum), but the disadvantages seem almost always to outweigh this meagre bonus. As exhibited in (1.12), our approach essentially yields a full exponential sum corresponding to the exceptional set in each mean value, whereas in (1.13) this exponential sum arises only once between two mean values, and hence has only half the impact on the ensuing analysis. This makes our results much harder to obtain, if not inaccessible, by the classical approach.

The flexibility and scope of the main method having been stressed in the preceding paragraphs, we end these introductory comments with the remark that further twists and turns may be introduced into the central technique. Such variants we intend to explore in forthcoming articles in this series. For example, one may investigate even $k$ th powers representable by sums of two primes, and establish an unconditional estimate for the corresponding exceptional set which is comparable to what was known hitherto under the assumption of the extended Riemann hypothesis for Dirichlet $L$-functions (see [3]). One can also modify the meaning of "exception". The general discussion above remains valid if the counting performed by $E_{\mathcal{B}}(N)$ refers to properties other than that the integer $n$ is not representable in the shape (1.4). To be more specific in the framework of Waring's problem for cubes, one may ask whether the asymptotic formula (1.1) holds for almost all $n$ when $n$ varies over the values of a quadratic polynomial. This is indeed the case when $s=6$.

THEOREM 1.3. - Let $\phi$ denote an integral quadratic polynomial, and let $D_{\phi}(N)$ denote the number of integers $n$ with $1 \leqslant n \leqslant N$ for which the asymptotic formula (1.1) fails for $R_{6}(\phi(n))$. Then $D_{\phi}(N) \ll N / \log N$.

We shall not prove this here but refer to another forthcoming article in this series [4] where amongst other things a stronger version of Theorem 1.3 will be presented. Finally, let it suffice to mention that there is also a variant of our methods which allows one to conclude, sometimes, that many (but not necessarily almost all) values of a thin sequence have a representation in a certain form. Limitations of space do not permit a more precise description of this and other variants, and we refer the interested reader to other articles in this series $[5,6]$.

Notation. Throughout, the letters $\varepsilon$ and $\eta$ will denote sufficiently small positive numbers. We take $P$ to be the basic parameter, a large real number depending at most on $\varepsilon, \eta$, and any coefficients of implicit polynomials if necessary. We use $\ll$ and $\gg$ to denote Vinogradov's 
well-known notation, implicit constants depending at most on $\varepsilon, \eta$ and implicit polynomials. Sometimes we make use of vector notation. For example, the expression $\left(c_{1}, \ldots, c_{t}\right)$ is abbreviated to c. Summations start at 1 unless indicated otherwise. In an effort to simplify our analysis, we adopt the following convention concerning the parameter $\varepsilon$. Whenever $\varepsilon$ appears in a statement, we assert that for each $\varepsilon>0$ the statement holds for sufficiently large values of the main parameter. Note that the "value" of $\varepsilon$ may consequently change from statement to statement, and hence also the dependence of implicit constants on $\varepsilon$.

\section{Exceptional sets for six cubes: the major arcs}

Our treatments of the exceptional sets arising from the representation of quadratic and cubic polynomials as sums of six cubes depend for their success on minor arc estimates of Brüdern [2]. Although the generating function corresponding to the six underlying cubes differs between the quadratic and cubic cases, there are sufficiently many common features that, at least so far as the major arcs are concerned, an essentially unified treatment is possible. The latter is the object of the present section.

Motivated by the notational conventions of Brüdern [2], we make the following definitions. We take $P$ to be a large real number, write $\eta$ for a sufficiently small positive number depending at most on $\varepsilon$, and consider a real number $R$ with $P^{\eta / 2}<R \leqslant P^{\eta}$. We write

$$
Q=P^{6 / 7}, \quad Y=P^{1 / 7},
$$

and define the generating functions

$$
f_{p}(\alpha)=\sum_{\substack{P<x \leqslant 2 P \\ p \nmid x}} e\left(\alpha x^{3}\right), \quad g(\alpha)=\sum_{Q<y \leqslant 2 Q} e\left(\alpha y^{3}\right), \quad h(\alpha)=\sum_{z \in \mathcal{A}(Q, R)} e\left(\alpha z^{3}\right),
$$

where $\mathcal{A}(Q, R)=\{1 \leqslant z \leqslant Q: p \mid z \Rightarrow p \leqslant R\}$. As a convenient substitute for the generating function central to the treatment of Brüdern [2], we define also

$$
\mathcal{F}(\alpha)=\sum_{\substack{Y<p \leqslant 2 Y \\ p \equiv 2}} f_{p}(\alpha) g\left(\alpha p^{3}\right) h\left(\alpha p^{3}\right)^{2}
$$

where the summation is over prime numbers. Finally, we define

$$
f(\alpha)=\sum_{P<x \leqslant 2 P} e\left(\alpha x^{3}\right), \quad t(\alpha)=\sum_{x \in \mathcal{A}(P, R)} e\left(\alpha x^{3}\right),
$$

and write

$$
\mathcal{S}_{2}(\alpha)=\mathcal{F}(\alpha) f(\alpha) t(\alpha), \quad \mathcal{S}_{3}(\alpha)=\mathcal{F}(\alpha) t(\alpha)^{2} .
$$

We remark that our proofs of Theorems 1.1 and 1.2 depend, respectively, on the use of the generating functions $\mathcal{S}_{2}(\alpha)$ and $\mathcal{S}_{3}(\alpha)$.

Next we define the major arcs central to the discussion of the present section. We write $L=(\log P)^{1 / 100}$, and define $\mathfrak{N}$ to be the union of the intervals

$$
\mathfrak{N}(q, a)=\left\{\alpha \in[0,1]:|q \alpha-a| \leqslant L P^{-3}\right\}
$$


with $0 \leqslant a \leqslant q \leqslant L$ and $(a, q)=1$. It is convenient at this stage to discuss the approximants to various generating functions on $\mathfrak{N}$. We define

$$
S(q, a)=\sum_{r=1}^{q} e\left(a r^{3} / q\right), \quad S(q, a, p)=S(q, a)-p^{-1} S\left(q, a p^{3}\right) .
$$

Notice that for $1 \leqslant q \leqslant L$ and $p>Y$ one has $p \nmid q$, and hence by a change of variables one obtains

$$
S\left(q, a p^{3}\right)=S(q, a)
$$

If we now define the multiplicative function $\kappa(q)$ on prime powers $\pi^{l}(l \in \mathbb{N})$ by means of the equations

$$
\kappa\left(\pi^{3 l}\right)=\pi^{-l}, \quad \kappa\left(\pi^{3 l+1}\right)=2 \pi^{-l-\frac{1}{2}}, \quad \kappa\left(\pi^{3 l+2}\right)=\pi^{-l-1},
$$

then it follows from Lemmata 4.3-4.5 of Vaughan [17] that whenever $p \equiv 2(\bmod 3)$ is a prime, and $q \in \mathbb{N}$ and $a \in \mathbb{Z}$ satisfy $(q, a)=1$, one has

$$
q^{-1}|S(q, a)| \ll \kappa(q), \quad q^{-1}|S(q, a, p)| \ll \kappa(q) .
$$

We note that the implicit constants in (2.8) are bounded above by 2 whenever $9 \nmid q$. We also define

$$
\begin{array}{ll}
v(\beta)=\int_{P}^{2 P} e\left(\beta \gamma^{3}\right) \mathrm{d} \gamma, & v_{1}(\beta)=\int_{0}^{P} e\left(\beta \gamma^{3}\right) \mathrm{d} \gamma, \\
w(\beta)=\int_{Q}^{2 Q} e\left(\beta \gamma^{3}\right) \mathrm{d} \gamma, & w_{1}(\beta)=\int_{0}^{Q} e\left(\beta \gamma^{3}\right) \mathrm{d} \gamma .
\end{array}
$$

Next we define the functions $f_{p}^{*}, g_{p}^{*}, t^{*}, f^{*}$ and $h_{p}^{*}$ for $\alpha \in[0,1]$ by taking

$$
\begin{gathered}
f_{p}^{*}(\alpha)=q^{-1} S(q, a, p) v(\alpha-a / q), \quad g_{p}^{*}(\alpha)=q^{-1} S\left(q, a p^{3}\right) w\left(p^{3}(\alpha-a / q)\right), \\
t^{*}(\alpha)=c_{\eta} q^{-1} S(q, a) v_{1}(\alpha-a / q), \quad h_{p}^{*}(\alpha)=c_{\eta} q^{-1} S\left(q, a p^{3}\right) w_{1}\left(p^{3}(\alpha-a / q)\right), \\
f^{*}(\alpha)=q^{-1} S(q, a) v(\alpha-a / q),
\end{gathered}
$$

when $\alpha \in \mathfrak{N}(q, a) \subset \mathfrak{N}$, and by taking each of these functions to be zero otherwise. Here we have written $c_{\eta}$ for $\rho\left(\eta^{-1}\right)$, where $\rho(t)$ is the Dickman function (see, for example, $\S 12.1$ of Vaughan [17]). It suffices for our purposes to note that when $\eta>0$ one has $c_{\eta}>0$. It follows from Theorem 4.1 of Vaughan [17] that for any $\alpha \in \mathbb{R}, a \in \mathbb{Z}$, and $q \in \mathbb{N}$, and for any prime $p \leqslant 2 Y$, one has

$$
\begin{gathered}
f_{p}(\alpha)-q^{-1} S(q, a, p) v(\alpha-a / q) \ll q^{\frac{1}{2}+\varepsilon}\left(1+P^{3}|\alpha-a / q|\right)^{1 / 2}, \\
g\left(\alpha p^{3}\right)-q^{-1} S\left(q, a p^{3}\right) w\left(p^{3}(\alpha-a / q)\right) \ll q^{\frac{1}{2}+\varepsilon}\left(1+P^{3}|\alpha-a / q|\right)^{1 / 2},
\end{gathered}
$$


and

$$
f(\alpha)-q^{-1} S(q, a) v(\alpha-a / q) \ll q^{\frac{1}{2}+\varepsilon}\left(1+P^{3}|\alpha-a / q|\right)^{1 / 2},
$$

whence for $\alpha \in \mathfrak{N}$ one has

$$
f_{p}(\alpha)-f_{p}^{*}(\alpha) \ll L^{\frac{1}{2}+\varepsilon}, \quad g\left(\alpha p^{3}\right)-g_{p}^{*}(\alpha) \ll L^{\frac{1}{2}+\varepsilon}, \quad f(\alpha)-f^{*}(\alpha) \ll L^{\frac{1}{2}+\varepsilon}
$$

Further, one may deduce from Lemma 8.5 of Wooley [20] (see also Lemma 5.4 of Vaughan [15] for a related conclusion) that for $\alpha \in \mathfrak{N}$ one has

$$
t(\alpha)-t^{*}(\alpha) \ll P(\log P)^{-1 / 4}, \quad h\left(\alpha p^{3}\right)-h_{p}^{*}(\alpha) \ll Q(\log P)^{-1 / 4} .
$$

Finally, by partial integration one readily confirms that the bounds

$$
\begin{gathered}
v(\beta) \ll P\left(1+P^{3}|\beta|\right)^{-1}, \quad w(\beta) \ll Q\left(1+Q^{3}|\beta|\right)^{-1}, \\
v_{1}(\beta) \ll P\left(1+P^{3}|\beta|\right)^{-1 / 3}, \quad
\end{gathered}
$$

hold uniformly for $\beta \in \mathbb{R}$.

LEMMA 2.1. - When $j=2$ or 3 , one has uniformly for $4 P^{3} \leqslant m \leqslant 64 P^{3}$ the estimate

$$
\int_{\mathfrak{N}} \mathcal{S}_{j}(\alpha) e(-\alpha m) \mathrm{d} \alpha \gg_{\eta} Y Q^{3}(\log P)^{-1}
$$

Proof. - The lower bound recorded in the lemma is a simple consequence of standard endgame technique in the circle method. We will be economical in our presentation of details, though with later applications in mind we work harder than is necessary for our immediate needs. We begin by writing

$$
\mathcal{S}_{j}^{*}(\alpha)=\sum_{\substack{Y<p \leqslant 2 Y \\ p \equiv 2(\bmod 3)}} f_{p}^{*}(\alpha) g_{p}^{*}(\alpha) h_{p}^{*}(\alpha)^{2} t^{*}(\alpha)^{j-1} f^{*}(\alpha)^{3-j} \quad(j=2,3) .
$$

Then it follows from (2.12) and (2.13) that for $\alpha \in \mathfrak{N}$ one has

$$
\mathcal{S}_{j}(\alpha)-\mathcal{S}_{j}^{*}(\alpha) \ll Y P^{3} Q^{3}(\log P)^{-5 / 4} .
$$

On recalling that the measure of $\mathfrak{N}$ is $\mathrm{O}\left(L^{2} P^{-3}\right)$, we deduce that

$$
\int_{\mathfrak{N}} \mathcal{S}_{j}(\alpha) e(-\alpha m) \mathrm{d} \alpha-\int_{\mathfrak{N}} \mathcal{S}_{j}^{*}(\alpha) e(-\alpha m) \mathrm{d} \alpha \ll Y Q^{3}(\log P)^{-6 / 5} .
$$

Next, on combining (2.16), (2.9)-(2.11), (2.5) and (2.6), we obtain

$$
\int_{\mathfrak{N}} \mathcal{S}_{j}^{*}(\alpha) e(-\alpha m) \mathrm{d} \alpha=\sum_{q \leqslant L} A(q, m) I_{j}^{*}\left(m ; L /\left(q P^{3}\right)\right),
$$


where

$$
A(q, m)=\sum_{\substack{a=1 \\(a, q)=1}}^{q} q^{-6} S(q, a)^{6} e(-a m / q)
$$

and, for each positive number $Z$,

$$
I_{j}^{*}(m ; Z)=c_{\eta}^{j+1} \sum_{\substack{Y<p \leqslant 2 Y \\ p \equiv 2}}\left(1-\frac{1}{p}\right) \int_{-Z}^{Z} V_{p}(\beta) e(-\beta m) \mathrm{d} \beta,
$$

where

$$
V_{p}(\beta)=v(\beta)^{4-j} v_{1}(\beta)^{j-1} w\left(p^{3} \beta\right) w_{1}\left(p^{3} \beta\right)^{2} .
$$

On writing

$$
I_{j}(m)=\lim _{Z \rightarrow \infty} I_{j}^{*}(m ; Z)
$$

we find from (2.14), (2.15) and (2.20) that for $0<\nu<\frac{1}{2}$ one has

$$
I_{j}(m)-I_{j}^{*}\left(m ; L /\left(q P^{3}\right)\right) \ll(q / L)^{\nu} Q^{3} Y(\log P)^{-1} .
$$

Then by (2.7), (2.8) and (2.19), we deduce that

$$
\begin{aligned}
\sum_{q \leqslant L}\left|A(q, m)\left(I_{j}(m)-I_{j}^{*}\left(m ; L /\left(q P^{3}\right)\right)\right)\right| & \ll Q^{3} Y L^{-\nu}(\log P)^{-1} \prod_{p}\left(1+64 p^{\nu-2}\right) \\
& \ll Q^{3} Y(\log P)^{-1-\nu / 100} .
\end{aligned}
$$

Moreover, also by (2.7), (2.8) and (2.19), we have

$$
\sum_{q>L}|A(q, m)| \ll \sum_{q=1}^{\infty}(q / L)^{\nu}|A(q, m)| \ll L^{-\nu} .
$$

Finally, by (2.14), (2.15) and (2.20) one finds that

$$
I_{j}(m) \ll Q^{3} Y(\log P)^{-1} .
$$

On combining (2.17), (2.18) and (2.21)-(2.23), we therefore conclude that

$$
\int_{\mathfrak{N}} \mathcal{S}_{j}(\alpha) e(-\alpha m) \mathrm{d} \alpha=\mathfrak{S}_{6}(m) I_{j}(m)+\mathrm{O}\left(Q^{3} Y(\log P)^{-1-\nu / 100}\right),
$$

where $\mathfrak{S}_{6}(m)$ is the singular series defined in connection with (1.1).

In order to complete the proof of the lemma we have only to note that a standard application of Fourier's integral formula (see, for example, Davenport [9]) shows that

$$
I_{j}(m) \gg Q^{3} Y(\log P)^{-1}
$$

for $4 P^{3} \leqslant m \leqslant 64 P^{3}$, and that Theorem 4.5 of Vaughan [17] demonstrates that $\mathfrak{S}_{6}(m) \gg 1$ uniformly in $m$. Consequently, the desired conclusion follows from (2.24). 
Equipped with Lemma 2.1, it is now a simple matter to describe our plan of attack on the theorems. Let $\phi_{j} \in \mathbb{Q}[t]$ be an integral polynomial with positive leading coefficient of degree $j$, where $j=2$ or 3 . We take

$$
6 P^{3}=\phi_{j}(N)
$$

and denote by $\mathcal{Z}_{j}(N)$ the set of integers $n$ with $N<n \leqslant 2 N$ for which the diophantine equation

$$
\phi_{j}(n)=x_{1}^{3}+x_{2}^{3}+\cdots+x_{6}^{3}
$$

has no solution in positive integers $x_{i}(1 \leqslant i \leqslant 6)$. Write

$$
K_{j}(\alpha)=\sum_{n \in \mathcal{Z}_{j}(N)} e\left(-\alpha \phi_{j}(n)\right) .
$$

Then it follows from the definition of $\mathcal{Z}_{j}(N)$ that

$$
\int_{0}^{1} \mathcal{S}_{j}(\alpha) K_{j}(\alpha) \mathrm{d} \alpha=0
$$

Next write $\mathfrak{n}=[0,1] \backslash \mathfrak{N}$. Then, on noting (2.25), it follows from Lemma 2.1 and (2.26) that

$$
\int_{\mathfrak{N}} \mathcal{S}_{j}(\alpha) K_{j}(\alpha) \mathrm{d} \alpha \gg \operatorname{card}\left(\mathcal{Z}_{j}(N)\right) Y Q^{3}(\log P)^{-1},
$$

whence by (2.27) we necessarily have

$$
\left|\int_{\mathfrak{n}} \mathcal{S}_{j}(\alpha) K_{j}(\alpha) \mathrm{d} \alpha\right| \gg \operatorname{card}\left(\mathcal{Z}_{j}(N)\right) Y Q^{3}(\log P)^{-1} .
$$

Our plan, which we execute in the next two sections, is to provide an upper bound for the integral on the left hand side of (2.28). As is apparent, an upper bound for $\operatorname{card}\left(\mathcal{Z}_{j}(N)\right)$ will follow immediately, and this will lead to the conclusions of Theorems 1.1 and 1.2.

\section{Values of quadratic polynomials which are not sums of six cubes}

In this section we complete the proof of Theorem 1.1. Large parts of the analysis of this section will be employed also in the next section wherein Theorem 1.2 will be established. We begin with a preparatory investigation of the block of four cubes lurking within the function $\mathcal{F}(\alpha)$. As might be expected, our minor arc treatment rests on a good estimate for the sixth moment of suitable cubic exponential sums.

LEMMA 3.1. - Let $U(X)$ denote the number of solutions of the diophantine equation

$$
x_{1}^{3}-x_{2}^{3}=y_{1}^{3}+y_{2}^{3}-y_{3}^{3}-y_{4}^{3},
$$

with $1 \leqslant x_{i} \leqslant 2 X(i=1,2)$ and $y_{j} \in \mathcal{A}\left(X, X^{\eta}\right)(1 \leqslant j \leqslant 4)$. Then provided that $\eta$ is sufficiently small, one has

$$
U(X) \ll X^{\frac{13}{4}-2 \eta}
$$


Proof. - The conclusion of the lemma follows from Theorem 1.2 of Wooley [22].

Henceforth we take $\eta$ to be a fixed positive number sufficiently small in the context of Lemma 3.1 and our ensuing argument. As an immediate application of Lemma 3.1, we note that by considering the underlying diophantine equations, it follows from (2.1) and (2.3) that

$$
\int_{0}^{1}\left|f(\alpha)^{2} t(\alpha)^{4}\right| \mathrm{d} \alpha \ll P^{\frac{13}{4}-2 \eta}, \quad \int_{0}^{1}\left|g(\alpha)^{2} h(\alpha)^{4}\right| \mathrm{d} \alpha \ll Q^{\frac{13}{4}-2 \eta} .
$$

The first of two lemmata concerning the block of exponential sums $\mathcal{F}(\alpha)$ is a simple modification of a conclusion contained in Brüdern [2], whereas the second is genuinely new. In order to describe these results we must first set up a Hardy-Littlewood dissection. When $X$ is a real number with $1 \leqslant X \leqslant P$, we define the set of major $\operatorname{arcs} \mathfrak{M}(X)$ to be the union of the intervals

$$
\mathfrak{M}(q, a)=\left\{\alpha \in[0,1]:|q \alpha-a| \leqslant X P^{-3}\right\}
$$

with $0 \leqslant a \leqslant q \leqslant X$ and $(a, q)=1$. We define $\mathfrak{m}(X)=[0,1] \backslash \mathfrak{M}(X)$ and for brevity write also

$$
\mathfrak{M}=\mathfrak{M}\left(P^{3 / 4}\right), \quad \mathfrak{m}=\mathfrak{m}\left(P^{3 / 4}\right) .
$$

We first explain how the work of Brüdern [2] leads to the estimate

$$
\int_{\mathfrak{m}(P)}|\mathcal{F}(\alpha)|^{2} \mathrm{~d} \alpha \ll Y^{2} Q^{6} P^{-19 / 14},
$$

where $\mathcal{F}(\alpha)$ is defined as in (2.2). Estimate (3.2) is essentially contained in the proof of Proposition 1 of Brïdern [2], but some comments are required in order that the reader is able easily to follow the necessary route. The mean value on the left hand side of (3.2) occurs explicitly in Eq. (3.2) of Brüdern [2], and is subsequently estimated therein to the precision claimed in (3.2), save for an extraneous factor of $P^{\varepsilon}$. We note that the sequence $\mathcal{A}$ used to define the exponential sum $h(\alpha)$ in Brüdern [2] differs from our set $\mathcal{A}(Q, R)$. However, the argument of Brüdern [2] does not depend on the specific shape of $\mathcal{A}$, and indeed it is only the mean value estimate (3.4) of Brüdern [2] which is vital, and such is available to us in the present context from (3.1). Moreover, the latter estimate is even a little stronger than the corresponding estimate (3.4) of Brüdern [2], and this additional strength may be carried through the argument to remove the offensive factor $P^{\varepsilon}$ implicit in Brüdern [2], Eq. (3.2), to which we alluded earlier.

Now define the functions $f_{p}^{*}(\alpha)$ and $g_{p}^{*}(\alpha)$ via (2.9) for $\alpha \in \mathfrak{M}(q, a) \subset \mathfrak{M}(P)$, and define these functions to be zero otherwise. Also write

$$
\mathcal{F}_{1}(\alpha)=\sum_{\substack{Y<p \leqslant 2 Y \\ p \equiv 2}} f_{p}^{*}(\alpha) g_{p}^{*}(\alpha) h\left(\alpha p^{3}\right)^{2} .
$$

Then an inspection of the proofs of inequalities (4.6) and (4.7) of Brüdern [2] reveals that

$$
\int_{\mathfrak{M}(P)}\left|\mathcal{F}(\alpha)-\mathcal{F}_{1}(\alpha)\right|^{2} \mathrm{~d} \alpha \ll Y^{2} Q^{6} P^{-19 / 14}
$$


Here again it is necessary to observe that the arguments of Brüdern [2] make use of the cubes underlying the exponential sum $h\left(\alpha p^{3}\right)$ only through upper bounds on the number of solutions of auxiliary diophantine equations, and consequently our alternative choice for $\mathcal{A}$ does not affect the argument.

Finally, we inspect the proof of Eq. (4.10) of Brüdern [2], but replace the choice $X=P^{\frac{4}{7}-\eta}$ therein with the new choice $X=P^{3 / 4}$. The work of $\S 6$ of Brüdern [2] then shows that inequality (4.10) of Brüdern [2] holds with $\eta=0$, whence we have

$$
\int_{\mathfrak{M}(P) \backslash \mathfrak{M}}\left|\mathcal{F}_{1}(\alpha)\right|^{2} \mathrm{~d} \alpha \ll Y^{2} Q^{6} P^{-19 / 14} .
$$

When combined with (3.2) and (3.4), we may summarise our deliberations thus far in a convenient form as follows.

LEMMA 3.2. - One has

$$
\int_{\mathfrak{m}}|\mathcal{F}(\alpha)|^{2} \mathrm{~d} \alpha \ll Y^{2} Q^{6} P^{-19 / 14}
$$

and

$$
\int_{\mathfrak{M}}\left|\mathcal{F}(\alpha)-\mathcal{F}_{1}(\alpha)\right|^{2} \mathrm{~d} \alpha \ll Y^{2} Q^{6} P^{-19 / 14} .
$$

Proof. - The first estimate follows in the manner indicated above, and the second is a trivial consequence of (3.4).

Our next lemma provides an upper bound for the contribution of $\mathcal{F}_{1}(\alpha)$ arising from the major arcs which is essentially best possible, and this estimate greatly facilitates our subsequent pruning procedures.

Lemma 3.3. - Suppose that $X$ is a real number with $1 \leqslant X \leqslant Q$. Then

$$
\int_{\mathfrak{M}(X)}\left|\mathcal{F}_{1}(\alpha)\right| \mathrm{d} \alpha \ll X^{\varepsilon} Y Q^{3} P^{-2}(\log Y)^{-1} .
$$

Proof. - Define $\kappa(q)$ via (2.7). Then, for each prime $p \in[Y, 2 Y]$ with $p \equiv 2(\bmod 3)$, and for each $\alpha \in \mathfrak{M}(q, a) \subset \mathfrak{M}(X)$, it follows from Eq. (6.5) of Brüdern [2] that

$$
f_{p}^{*}(\alpha) g_{p}^{*}(\alpha) \ll \kappa(q)^{2} P Q\left(1+P^{3}|\alpha-a / q|\right)^{-2},
$$

and moreover that when $p^{2} \mid q$, one has

$$
f_{p}^{*}(\alpha) g_{p}^{*}(\alpha)=0
$$

On recalling (3.3), we therefore deduce that

$$
\int_{\mathfrak{M}(X)}\left|\mathcal{F}_{1}(\alpha)\right| \mathrm{d} \alpha \ll P Q \sum_{q \leqslant X} \kappa(q)^{2} \sum_{\substack{Y<p \leqslant 2 Y \\ p^{2} \nmid q}} \int_{-\infty}^{\infty} \sum_{\substack{a=1 \\(a, q)=1}}^{q} \frac{\left|h\left(p^{3}(a / q+\beta)\right)\right|^{2}}{\left(1+P^{3}|\beta|\right)^{2}} \mathrm{~d} \beta
$$


Let $c_{q}(h)$ be Ramanujan's sum, which we define by

$$
c_{q}(h)=\sum_{\substack{a=1 \\(a, q)=1}}^{q} e(a h / q) .
$$

Then it follows that

$$
\sum_{\substack{a=1 \\(a, q)=1}}^{q}\left|h\left(p^{3}(a / q+\beta)\right)\right|^{2}=\sum_{x, y \in \mathcal{A}(Q, R)} c_{q}\left(p^{3}\left(x^{3}-y^{3}\right)\right) e\left(\beta p^{3}\left(x^{3}-y^{3}\right)\right) .
$$

The familiar estimate $\left|c_{q}(h)\right| \leqslant(q, h)$ (noting the convention that $\left.(q, 0)=q\right)$ therefore leads from (3.5) to the estimate

$$
\int_{\mathfrak{M}(X)}\left|\mathcal{F}_{1}(\alpha)\right| \mathrm{d} \alpha \ll P^{-2} Q \sum_{q \leqslant X} \kappa(q)^{2} \sum_{\substack{Y<p \leqslant 2 Y \\ p^{2} \nmid q}} \sum_{1 \leqslant x, y \leqslant Q}\left(q, p^{3}\left(x^{3}-y^{3}\right)\right) .
$$

When $p^{2} \nmid q$, one plainly has

$$
\left(q, p^{3}\left(x^{3}-y^{3}\right)\right) \leqslant(p, q)\left(q, x^{3}-y^{3}\right) .
$$

Observe that when $q \leqslant X, p>Y$ and $X \leqslant Y$ one has $(p, q)=1$. Meanwhile, when $X>Y$ one has $\log Y \ll X^{\varepsilon / 2}$. Thus, on applying a familiar estimate for the divisor function, it follows that whenever $1 \leqslant q \leqslant X$, one has

$$
\sum_{Y<p \leqslant 2 Y}(p, q) \ll \frac{Y}{\log Y}+\frac{X^{\varepsilon / 2}}{\log Y} \sum_{\substack{p \mid q \\ Y<p \leqslant 2 Y}} p \ll \frac{X^{\varepsilon} Y}{\log Y}
$$

On combining (3.6) and (3.7), we therefore deduce that

$$
\int_{\mathfrak{M}(X)}\left|\mathcal{F}_{1}(\alpha)\right| \mathrm{d} \alpha \ll X^{\varepsilon} P^{-2} Q Y(\log Y)^{-1} \sum_{q \leqslant X} \kappa(q)^{2} \sum_{1 \leqslant x, y \leqslant Q}\left(q, x^{3}-y^{3}\right) .
$$

Next write $\rho(d)$ for the number of solutions of the congruence $x^{3} \equiv y^{3}(\bmod d)$ with $1 \leqslant x, y \leqslant d$. Then, by sorting $x$ and $y$ into residue classes modulo $d$, it follows that whenever $q \leqslant Q$

$$
\begin{aligned}
\sum_{1 \leqslant x, y \leqslant Q}\left(q, x^{3}-y^{3}\right) & \leqslant \sum_{d \mid q} d \operatorname{card}\left\{1 \leqslant x, y \leqslant Q: x^{3} \equiv y^{3}(\bmod d)\right\} \\
& \leqslant \sum_{d \mid q}\left(\frac{Q}{d}+1\right)^{2} d \rho(d) \ll Q^{2} \sum_{d \mid q} \frac{\rho(d)}{d} .
\end{aligned}
$$

For any natural number $r$ one may write $r=r_{1} r_{3}^{3}$ with $r_{1}$ cube-free, and this decomposition is unique. Further, an elementary counting argument shows that $\rho(r) \ll r^{1+\varepsilon} r_{3}$. Consequently, on 
recalling (3.9),

$$
\sum_{1 \leqslant x, y \leqslant Q}\left(q, x^{3}-y^{3}\right) \ll Q^{2} q^{\varepsilon} q_{3}
$$

But in view of (2.7) we have $\kappa(q) \ll q^{\varepsilon} q_{1}^{-1 / 2} q_{3}^{-1}$, whence

$$
\sum_{q \leqslant X} \kappa(q)^{2} q_{3} \ll X^{\varepsilon / 2} \sum_{q_{1} q_{3}^{3} \leqslant X} q_{1}^{-1} q_{3}^{-1} \ll X^{\varepsilon} .
$$

On recalling (3.8) and (3.10), therefore, the proof of the lemma is complete.

We require a variant of Lemma 3.3 of simpler type in order to complete the proof of Theorem 1.1.

LEMMA 3.4. - One has

$$
\int_{\mathfrak{M}}|f(\alpha) t(\alpha)|^{2} \mathrm{~d} \alpha \ll P^{1+\varepsilon}
$$

Proof. - When $1 \leqslant q \leqslant P^{3 / 4}$ and $|q \alpha-a| \leqslant P^{-9 / 4}$, it follows from (2.8), (2.14) together with Theorem 4.1 of Vaughan [17] that

$$
f(\alpha) \ll \kappa(q) P\left(1+P^{3}|\alpha-a / q|\right)^{-1}+q^{\frac{1}{2}+\varepsilon} .
$$

Since it follows readily from (2.7) that $\kappa(q) \gg q^{-1 / 2}$, it is easily confirmed that the first term on the right hand side of (3.11) always dominates the second. Consequently,

$$
\int_{\mathfrak{M}}|f(\alpha) t(\alpha)|^{2} \mathrm{~d} \alpha \ll P^{2} \sum_{q \leqslant P^{3 / 4}} \kappa(q)^{2} \int_{-\infty}^{\infty} \sum_{\substack{a=1 \\(a, q)=1}}^{q}|t(a / q+\beta)|^{2}\left(1+P^{3}|\beta|\right)^{-2} \mathrm{~d} \beta
$$

and a comparison with the expression (3.5) will convince the reader that the proof of the present lemma may be completed by applying the argument of the proof of the previous one, mutatis mutandis.

We pause before launching our campaign proper to add a further simple estimate to our arsenal of mean values. By employing Weyl's inequality in the form given by Vaughan [14], Lemma 1, one has

$$
\sup _{\alpha \in \mathfrak{m}}|f(\alpha)| \ll P^{\frac{3}{4}+\varepsilon},
$$

and in combination with (3.1) we obtain the estimate

$$
\int_{\mathfrak{m}}|f(\alpha) t(\alpha)|^{4} \mathrm{~d} \alpha \ll P^{\frac{19}{4}-\eta} .
$$

Our starting point for the proof of Theorem 1.1 is the lower bound (2.28). As a first step in obtaining a corresponding upper bound we remove the contribution to the integral on the left hand side of (2.28) arising from the set $\mathfrak{n} \cap \mathfrak{M}$. Note first that $\mathfrak{N}=\mathfrak{M}(L)$, and so $\mathfrak{n} \cap \mathfrak{M}$ is contained in the union of the sets

$$
\mathfrak{K}(X)=\mathfrak{M}(2 X) \backslash \mathfrak{M}(X),
$$


where we put $X=2^{l} L$, and take the union with $l \geqslant 0$ satisfying $2^{l} L \leqslant P^{3 / 4}$. Next, on recalling (2.7) and (3.11), we find that for $X \leqslant P^{3 / 4}$, one has

$$
\sup _{\alpha \in \mathfrak{K}(X)}|f(\alpha)| \ll P X^{-1 / 3} .
$$

Consequently, on making use of the trivial bound $|t(\alpha)| \leqslant P$, we infer from Lemma 3.3 that for $X \leqslant P^{3 / 4}$ one has

$$
\int_{\mathfrak{K}(X)}\left|\mathcal{F}_{1}(\alpha) f(\alpha) t(\alpha)\right| \mathrm{d} \alpha \ll P^{2} X^{-1 / 3} \int_{\mathfrak{M}(2 X)}\left|\mathcal{F}_{1}(\alpha)\right| \mathrm{d} \alpha \ll Q^{3} Y X^{-1 / 4}(\log Y)^{-1} .
$$

On summing over the aforementioned values of $l$, we find that the total contribution arising from the union of the $\operatorname{arcs} \mathfrak{K}\left(2^{l} L\right)$ yields the upper bound

$$
\int_{\mathfrak{n} \cap \mathfrak{M}}\left|\mathcal{F}_{1}(\alpha) f(\alpha) t(\alpha)\right| \mathrm{d} \alpha \ll Q^{3} Y L^{-1 / 4}(\log Y)^{-1} .
$$

Next, applying Schwarz's inequality in combination with Lemmata 3.2 and 3.4, we obtain

$$
\begin{aligned}
\int_{\mathfrak{M}} & \left|\left(\mathcal{F}(\alpha)-\mathcal{F}_{1}(\alpha)\right) f(\alpha) t(\alpha)\right| \mathrm{d} \alpha \\
\leqslant & \left(\int_{\mathfrak{M}}\left|\mathcal{F}(\alpha)-\mathcal{F}_{1}(\alpha)\right|^{2} \mathrm{~d} \alpha\right)^{1 / 2}\left(\int_{\mathfrak{M}}|f(\alpha) t(\alpha)|^{2} \mathrm{~d} \alpha\right)^{1 / 2} \\
& \ll\left(Y^{2} Q^{6} P^{-19 / 14}\right)^{\frac{1}{2}}\left(P^{1+\varepsilon}\right)^{\frac{1}{2}} \ll Q^{3} Y P^{-1 / 6} .
\end{aligned}
$$

We therefore find from (3.13) and (2.4) that

$$
\int_{\mathfrak{n} \cap \mathfrak{M}}\left|\mathcal{S}_{2}(\alpha)\right| \mathrm{d} \alpha \ll Q^{3} Y L^{-1 / 4}(\log Y)^{-1}
$$

whence the trivial bound $\left|K_{2}(\alpha)\right| \leqslant \operatorname{card} \mathcal{Z}_{2}(N)$, in combination with (2.28), reveals that

$$
\int_{\mathfrak{m}}\left|\mathcal{S}_{2}(\alpha) K_{2}(\alpha)\right| \mathrm{d} \alpha \gg Y Q^{3}(\log P)^{-1} \operatorname{card} \mathcal{Z}_{2}(N) .
$$

We provide an upper bound for the mean value on the left hand side of (3.14) via Hölder's inequality, obtaining from (2.4) the estimate

$$
\int_{\mathfrak{m}}\left|\mathcal{S}_{2}(\alpha) K_{2}(\alpha)\right| \mathrm{d} \alpha \leqslant J^{1 / 4}\left(\int_{\mathfrak{m}}|\mathcal{F}(\alpha)|^{2} \mathrm{~d} \alpha\right)^{1 / 2}\left(\int_{\mathfrak{m}}|f(\alpha) t(\alpha)|^{4} \mathrm{~d} \alpha\right)^{1 / 4},
$$

where

$$
J=\int_{0}^{1}\left|K_{2}(\alpha)\right|^{4} \mathrm{~d} \alpha
$$


But on considering the underlying diophantine equation, one finds that $J$ is equal to the number of solutions of the equation

$$
\phi_{2}\left(n_{1}\right)+\phi_{2}\left(n_{2}\right)=\phi_{2}\left(n_{3}\right)+\phi_{2}\left(n_{4}\right)
$$

with $n_{j} \in \mathcal{Z}_{2}(N)(1 \leqslant j \leqslant 4)$. By completing the square in the quadratic polynomial $\phi_{2}(t)$, one recognises easily that it suffices to bound the number $J_{0}(\mathcal{Z})$ of solutions of the simpler equation $z_{1}^{2}+z_{2}^{2}=z_{3}^{2}+z_{4}^{2}$, with $z_{i} \in \mathcal{Z}(1 \leqslant i \leqslant 4)$, in which $\mathcal{Z}$ is a set of integers of the same cardinality as $\mathcal{Z}_{2}(N)$. On separating diagonal solutions, an elementary estimate for the divisor function readily shows that

$$
J \ll P^{\varepsilon}\left(\operatorname{card} \mathcal{Z}_{2}(N)\right)^{2} .
$$

Then by (3.15), (3.16), Lemma 3.2 and the upper bound (3.12), we derive the estimate

$$
\int_{\mathfrak{m}}\left|\mathcal{S}_{2}(\alpha) K_{2}(\alpha)\right| \mathrm{d} \alpha \ll\left(\operatorname{card} \mathcal{Z}_{2}(N)\right)^{\frac{1}{2}} Y Q^{3} P^{\frac{57}{112}-\frac{1}{5} \eta} .
$$

The proof of Theorem 1.1 is now readily completed. On combining (3.14), (3.17) and (2.25), we obtain

$$
\operatorname{card} \mathcal{Z}_{2}(N) \ll P^{57 / 56} \ll N^{19 / 28},
$$

and the desired conclusion follows by summing over dyadic intervals.

\section{Values of cubic polynomials which are not sums of six cubes}

Although the treatment of quadratic polynomials in the previous section provides many of the tools required in the application of our methods in the cubic situation, in order to achieve the bound claimed in Theorem 1.2 we require still more sophisticated weaponry. Much of this section will be devoted to the proof of a mean value estimate based on efficient differencing with certain variables restricted to thin sets.

Before describing our differencing lemma we require some additional exponential sums to be defined. We first extend the definition (2.3), writing for each positive number $U$,

$$
t(\alpha ; U)=\sum_{x \in \mathcal{A}(U, R)} e\left(\alpha x^{3}\right)
$$

We take $\theta$ to be a parameter with $0<\theta<\frac{1}{3}$, to be chosen later, and write

$$
M=P^{\theta}, \quad Q_{1}=P M^{-1}, \quad H=N M^{-3} .
$$

Here we recall that in the present circumstance wherein $j=3$, Eq. (2.25) ensures that $N \asymp P$. Finally, we write

$$
G_{1}(\alpha)=\sum_{M<m \leqslant M R} \sum_{h \leqslant N / m^{3}} \sum_{\substack{z \in \mathcal{Z}_{3}(N) \\ z+h m^{3} \in \mathcal{Z}_{3}(N)}} e\left(\alpha m^{-3}\left(\phi_{3}\left(z+h m^{3}\right)-\phi_{3}(z)\right)\right) .
$$


LEMMA 4.1. - For each positive number $\varepsilon$, one has

$$
\int_{0}^{1}\left|K_{3}(\alpha)^{2} t(\alpha ; P)^{4}\right| \mathrm{d} \alpha \ll P^{2+\varepsilon} M^{2} R^{4} \operatorname{card} \mathcal{Z}_{3}(N)+M^{3+\varepsilon} R^{3} \int_{0}^{1}\left|G_{1}(\alpha) t\left(\alpha ; Q_{1}\right)^{4}\right| \mathrm{d} \alpha .
$$

Proof. - On considering the underlying diophantine equations, an inspection of the proof of Lemma 2.2 of Wooley [21] reveals that with trivial modifications, the latter argument establishes the upper bound

$$
\int_{0}^{1}\left|K_{3}(\alpha)^{2} t(\alpha ; P)^{4}\right| \mathrm{d} \alpha \ll P^{\varepsilon}\left(S_{1}+P M S_{2}+(M R)^{3} S_{3}\right),
$$

where

$$
S_{1}=\int_{0}^{1}\left|K_{3}(\alpha)^{2} t(\alpha ; M)^{4}\right| \mathrm{d} \alpha
$$

$$
S_{2}=\int_{0}^{1}\left|K_{3}(\alpha)^{2} t(\alpha ; P)^{2}\right| \mathrm{d} \alpha
$$

and $S_{3}$ denotes the number of solutions of the diophantine equation

$$
\phi_{3}\left(z_{1}\right)+w^{3}\left(u_{1}^{3}+u_{2}^{3}\right)=\phi_{3}\left(z_{2}\right)+w^{3}\left(u_{3}^{3}+u_{4}^{3}\right)
$$

with

$$
\begin{gathered}
M<w \leqslant M R, \quad u_{j} \in \mathcal{A}\left(Q_{1}, R\right), \\
z_{1}, z_{2} \in \mathcal{Z}_{3}(N), \quad z_{1} \equiv z_{2}\left(\bmod w^{3}\right) .
\end{gathered}
$$

Observe first that the solutions of (4.7) counted by $S_{3}$ satisfying $z_{1}=z_{2}, u_{1}=u_{3}$ and $u_{2}=u_{4}$ number

$$
\gg M(P / M)^{2} \operatorname{card} \mathcal{Z}_{3}(N)
$$

whence

$$
(M R)^{3} S_{3} \gg P^{2} M^{2} R^{3} \operatorname{card} \mathcal{Z}_{3}(N) .
$$

Next, on estimating the exponential sum $t(\alpha ; M)$ trivially via (4.1), we find from (4.5) that

$$
S_{1} \leqslant M^{4} \int_{0}^{1}\left|K_{3}(\alpha)\right|^{2} \mathrm{~d} \alpha \leqslant M^{4} \operatorname{card} \mathcal{Z}_{3}(N)
$$

by Parseval's identity, whence by making use of our hypothesis that $M^{3} \leqslant P$, one has

$$
S_{1} \leqslant P M \operatorname{card} \mathcal{Z}_{3}(N) \ll(M R)^{3} S_{3} .
$$


Finally, it follows from (4.6) by orthogonality that $S_{2}$ is equal to the number of solutions of the diophantine equation

$$
\phi_{3}\left(z_{1}\right)-\phi_{3}\left(z_{2}\right)=v_{1}^{3}-v_{2}^{3},
$$

with $z_{1}, z_{2} \in \mathcal{Z}_{3}(N)$ and $v_{1}, v_{2} \in \mathcal{A}(P, R)$. The number of such solutions of (4.12) with $z_{1}=z_{2}$ is plainly $\mathrm{O}\left(P\left(\operatorname{card} \mathcal{Z}_{3}(N)\right)\right)$. Fix a choice of $z_{1}, z_{2} \in \mathcal{Z}_{3}(N)$ with $z_{1} \neq z_{2}$, and consider a solution $v_{1}, v_{2}$ of (4.12). Since $v_{1}-v_{2}$ is a divisor of the non-zero integer $\phi_{3}\left(z_{1}\right)-\phi_{3}\left(z_{2}\right)$, it follows from an elementary estimate for the divisor function that the number of possible choices for $v_{1}-v_{2}$ is $\mathrm{O}\left(P^{\varepsilon}\right)$. But given a fixed choice of $v_{1}-v_{2}$, one finds that $v_{1}+v_{2}$ is determined essentially uniquely by (4.12), whence also $v_{1}, v_{2}$. Thus we conclude that the number of solutions of (4.12) counted implicitly by $S_{2}$ with $z_{1} \neq z_{2}$ is at most $\mathrm{O}\left(P^{\varepsilon}\left(\operatorname{card} \mathcal{Z}_{3}(N)\right)^{2}\right)$. Consequently,

$$
S_{2} \ll P \operatorname{card} \mathcal{Z}_{3}(N)+P^{\varepsilon}\left(\operatorname{card} \mathcal{Z}_{3}(N)\right)^{2} \ll P^{1+\varepsilon} \operatorname{card} \mathcal{Z}_{3}(N),
$$

whence by (4.10),

$$
P M S_{2} \ll P^{2+\varepsilon} M \operatorname{card} \mathcal{Z}_{3}(N) \ll P^{\varepsilon}(M R)^{3} S_{3} .
$$

On combining (4.4), (4.11) and (4.13) we may conclude thus far that

$$
\int_{0}^{1}\left|K_{3}(\alpha)^{2} t(\alpha ; P)^{4}\right| \mathrm{d} \alpha \ll P^{\varepsilon}(M R)^{3} S_{3} .
$$

Next we establish an upper bound for $S_{3}$. Observe that when $z_{1}$ and $z_{2}$ are elements of $\mathcal{Z}_{3}(N)$ satisfying (4.9) and $z_{1}>z_{2}$, then one may write $z_{2}=z$ and $z_{1}=z+h w^{3}$ with $z \in \mathcal{Z}_{3}(N)$, $1 \leqslant h \leqslant N / w^{3}$ and $M<w \leqslant M R$. Then, by isolating the diagonal contribution with $z_{1}=z_{2}$ in (4.7), and considering the underlying diophantine equations, one finds from (4.8) and (4.3) that

$$
S_{3} \ll S_{4}+S_{5},
$$

where

$$
S_{4}=M R\left(\operatorname{card} \mathcal{Z}_{3}(N)\right) \int_{0}^{1}\left|t\left(\alpha ; Q_{1}\right)\right|^{4} \mathrm{~d} \alpha
$$

and

$$
S_{5}=\int_{0}^{1}\left|G_{1}(\alpha) t\left(\alpha ; Q_{1}\right)^{4}\right| \mathrm{d} \alpha .
$$

But it follows from Hua's Lemma (see, for example, Vaughan [17], Lemma 2.5) that

$$
\int_{0}^{1}\left|t\left(\alpha ; Q_{1}\right)\right|^{4} \mathrm{~d} \alpha \ll Q_{1}^{2+\varepsilon},
$$

whence by (4.16) one has

$$
S_{4} \ll\left(\operatorname{card} \mathcal{Z}_{3}(N)\right) M R Q_{1}^{2+\varepsilon}
$$


Thus, collecting together (4.14), (4.15), (4.17) and (4.18), and recalling (4.2), we complete the proof of the lemma.

Our understanding of the behaviour of the exponential sum $G_{1}(\alpha)$ is weak, since the latter depends heavily on the set $\mathcal{Z}_{3}(N)$. We therefore engineer a metamorphosis which facilitates our subsequent analysis. To be precise at this stage we must be explicit regarding the polynomial $\phi_{3}$. We write the latter in the shape

$$
\phi_{3}(t)=\Delta^{-1}\left(a t^{3}+b t^{2}+c t+d\right)
$$

for suitable integers $a, b, c, d, \Delta$. Write also $A=12 a \Delta$ and $B=12 a c-4 b^{2}$. Further, we define the exponential sums $F_{1}(\alpha)$ and $\tilde{G}_{1}(\alpha)$ associated with $\phi_{3}$ by

$$
F_{1}(\alpha)=\sum_{M<m \leqslant M} \sum_{h \leqslant H} \sum_{y \leqslant 2 A N} e\left(\alpha h\left(y^{2}+3 a^{2} h^{2} m^{6}+B\right)\right)
$$

and

$$
\tilde{G}_{1}(\alpha)=\sum_{M<m \leqslant M R} \sum_{h \leqslant H} \sum_{z \in \mathcal{Z}_{3}(N)} e\left(\alpha m^{-3}\left(\phi_{3}\left(z+h m^{3}\right)-\phi_{3}(z)\right)\right) .
$$

Finally, we define the mean value

$$
\mathcal{J}=\int_{0}^{1}\left|F_{1}(\alpha) \tilde{G}_{1}(A \alpha) t\left(A \alpha ; Q_{1}\right)^{2}\right| \mathrm{d} \alpha .
$$

LEMMA 4.2. - One has

$$
\int_{0}^{1}\left|G_{1}(\alpha) t\left(\alpha ; Q_{1}\right)^{4}\right| \mathrm{d} \alpha \ll \mathcal{J}^{1 / 2}\left(\int_{0}^{1}\left|t\left(\alpha ; Q_{1}\right)\right|^{6} \mathrm{~d} \alpha\right)^{1 / 2} .
$$

Proof. - By Schwarz's inequality, one has

$$
\int_{0}^{1}\left|G_{1}(\alpha) t\left(\alpha ; Q_{1}\right)^{4}\right| \mathrm{d} \alpha \ll \mathcal{J}_{1}^{1 / 2}\left(\int_{0}^{1}\left|t\left(\alpha ; Q_{1}\right)\right|^{6} \mathrm{~d} \alpha\right)^{1 / 2}
$$

where

$$
\mathcal{J}_{1}=\int_{0}^{1}\left|G_{1}(\alpha)^{2} t\left(\alpha ; Q_{1}\right)^{2}\right| \mathrm{d} \alpha .
$$

It is evident, therefore, that the lemma will follow from the bound $\mathcal{J}_{1} \ll \mathcal{J}$ which we now prove. Observe first that by (4.19) one has

$$
m^{-3}\left(\phi_{3}\left(z+h m^{3}\right)-\phi_{3}(z)\right)=\frac{h}{12 a \Delta}\left(\left(3 a\left(2 z+h m^{3}\right)+2 b\right)^{2}+3 a^{2} h^{2} m^{6}+\left(12 a c-4 b^{2}\right)\right) .
$$


Then on considering the underlying diophantine equation, it follows from (4.23) that $\mathcal{J}_{1}$ is bounded above by the number of integral solutions of the equation

$$
m_{1}^{-3}\left(\phi_{3}\left(z+h_{1} m_{1}^{3}\right)-\phi_{3}(z)\right)-\frac{h_{2}}{12 a \Delta}\left(y^{2}+3 a^{2} h_{2}^{2} m_{2}^{6}+B\right)=u_{1}^{3}-u_{2}^{3}
$$

with

$$
\begin{gathered}
u_{1}, u_{2} \in \mathcal{A}\left(Q_{1}, R\right), \quad M<m_{1}, m_{2} \leqslant M R, \quad z \in \mathcal{Z}_{3}(N), \\
1 \leqslant h_{1}, h_{2} \leqslant H, \quad 1 \leqslant y \leqslant 2 A N,
\end{gathered}
$$

and satisfying the property that $12 a \Delta \mid h_{2}\left(y^{2}+3 a^{2} h_{2}^{2} m_{2}^{6}+B\right)$. On recalling definitions (4.1), (4.20), (4.21), and considering the underlying diophantine equation, it therefore follows from orthogonality that

$$
\mathcal{J}_{1} \ll \int_{0}^{1} F_{1}(-\alpha) \tilde{G}_{1}(A \alpha)\left|t\left(A \alpha ; Q_{1}\right)\right|^{2} \mathrm{~d} \alpha,
$$

and the desired conclusion that $\mathcal{J}_{1} \ll \mathcal{J}$ is immediate from (4.22).

We estimate the mean value $\mathcal{J}$ by using the Hardy-Littlewood method, but this will entail some preparation. We define the major arcs $\mathfrak{P}$ to be the union of the intervals

$$
\mathfrak{P}(q, a)=\left\{\alpha \in[0,1]:|q \alpha-a| \leqslant N Q_{1}^{-3}\right\}
$$

with $0 \leqslant a \leqslant q \leqslant N$ and $(a, q)=1$. We then write $\mathfrak{p}=[0,1] \backslash \mathfrak{P}$ for the minor arcs. The analysis of the minor arcs requires several auxiliary mean value estimates, which we presently discuss.

LEMMA 4.3. - One has

$$
\int_{0}^{1}\left|F_{1}(\alpha)\right|^{2} \mathrm{~d} \alpha \ll N^{1+\varepsilon} H M R
$$

and

$$
\int_{0}^{1}\left|\tilde{G}_{1}(\alpha)\right|^{2} \mathrm{~d} \alpha \ll N^{\varepsilon} H M R \operatorname{card} \mathcal{Z}_{3}(N) .
$$

Proof. - On recalling (4.20) it follows from orthogonality that the mean value (4.24) is equal to the number of integral solutions of the equation

$$
h_{1}\left(y_{1}^{2}+3\left(a h_{1} m_{1}^{3}\right)^{2}+B\right)=h_{2}\left(y_{2}^{2}+3\left(a h_{2} m_{2}^{3}\right)^{2}+B\right),
$$

with

$$
M<m_{1}, m_{2} \leqslant M R, \quad 1 \leqslant h_{1}, h_{2} \leqslant H, \quad 1 \leqslant y_{1}, y_{2} \leqslant 2 A N .
$$

Observe that given a fixed choice of $y_{2}, h_{2}, m_{2}$ satisfying (4.27), one has

$$
\left(a h_{2} m_{2}^{3}\right)^{2} \gg M^{6}>|B|
$$


and thus the right hand side of (4.26) is a non-zero integer $l$. It follows that there are at most $\mathrm{O}\left(N^{\varepsilon}\right)$ possible choices for $y_{1}, h_{1}, m_{1}$ satisfying (4.26) and (4.27). For $h_{1}$ is a divisor of $l$, and an elementary estimate for the divisor function shows that there are at most $\mathrm{O}\left(l^{\varepsilon}\right)$ such. Fix any one such choice, and consider the equation

$$
u^{2}+3 v^{2}=\frac{l}{h_{1}}-B
$$

Familiar estimates for the number of representations of an integer by a binary quadratic form (see, for example, Estermann [10]) show that the number of solutions of the latter equation in $u$ and $v$ is $\mathrm{O}\left(\left(l / h_{1}-B\right)^{\varepsilon}\right)$ if $l / h_{1}-B \geqslant 0$, and zero otherwise. Our earlier assertion now follows. Thus the number of solutions of (4.26) satisfying (4.27) is $\mathrm{O}\left(N^{1+\varepsilon} H M R\right)$. This completes the proof of (4.24).

We treat the mean value (4.25) in a similar fashion. On recalling (4.21) and the argument of the proof of Lemma 4.2, we find that the mean value (4.25) is bounded above by the number of integral solutions of the equation

$$
\begin{aligned}
& h_{1}\left(\left(3 a\left(2 z_{1}+h_{1} m_{1}^{3}\right)+2 b\right)^{2}+3\left(a h_{1} m_{1}^{3}\right)^{2}+B\right) \\
& \quad=h_{2}\left(\left(3 a\left(2 z_{2}+h_{2} m_{2}^{3}\right)+2 b\right)^{2}+3\left(a h_{2} m_{2}^{3}\right)^{2}+B\right)
\end{aligned}
$$

with

$$
M<m_{1}, m_{2} \leqslant M R, \quad 1 \leqslant h_{1}, h_{2} \leqslant H, \quad z_{1}, z_{2} \in \mathcal{Z}_{3}(N) .
$$

Given a fixed choice of $z_{2}, h_{2}, m_{2}$ satisfying (4.29), it again follows that the right hand side of (4.28) is a non-zero integer. A comparison between Eqs. (4.26) and (4.28) reveals that one may apply the argument of the preceding paragraph to show that there are at most $\mathrm{O}\left(N^{\varepsilon}\right)$ possible choices for $z_{1}, h_{1}, m_{1}$ satisfying (4.28) and (4.29). Thus the number of solutions of (4.28) and (4.29) is

$$
\ll N^{\varepsilon} H M R \operatorname{card} \mathcal{Z}_{3}(N) .
$$

The estimate (4.25) follows immediately.

One may provide an estimate for the sum $F_{1}(\alpha)$ mirroring bounds for analogous sums provided by Vaughan [14], [15]. By Cauchy's inequality, one has

$$
\left|F_{1}(\alpha)\right|^{2} \leqslant D(\alpha) E(\alpha),
$$

where

$$
D(\alpha)=\sum_{h \leqslant H}\left|\sum_{y \leqslant 2 A N} e\left(\alpha h y^{2}\right)\right|^{2}
$$

and

$$
E(\alpha)=\sum_{h \leqslant H}\left|\sum_{M<m \leqslant M R} e\left(3 a^{2} \alpha h^{3} m^{6}\right)\right|^{2} .
$$

LEMMA 4.4. - One has

$$
\sup _{\alpha \in \mathfrak{p}}|D(\alpha)| \ll N^{1+\varepsilon} H .
$$


Moreover, when $a \in \mathbb{Z}, q \in \mathbb{N},(a, q)=1$ and $\alpha \in \mathfrak{P}(q, a) \subset \mathfrak{P}$, one has

$$
D(\alpha) \ll N^{2+\varepsilon} H\left(q+Q_{1}^{3}|q \alpha-a|\right)^{-1} .
$$

Proof. - The conclusions of the lemma are immediate consequences of the argument of the proof of Lemma 3.1 of Vaughan [15].

Rather than follow the trail laid down by Vaughan [15] in our analysis of $E(\alpha)$ we instead make use of mean value estimates which lead to sharper conclusions. In this context we define the exponential sum

$$
E^{*}(\alpha)=\sum_{h \leqslant H} \sum_{M<m_{2}<m_{1} \leqslant M R} e\left(\alpha h^{3}\left(m_{1}^{6}-m_{2}^{6}\right)\right),
$$

and note that by (4.31) one has

$$
E(\alpha) \ll H M R+\left|E^{*}\left(3 a^{2} \alpha\right)\right| .
$$

LEMMA 4.5. - When $j=1$ or 2 , one has

$$
\int_{0}^{1}\left|E^{*}(\alpha)\right|^{2^{j}} \mathrm{~d} \alpha \ll P^{\varepsilon} H^{2^{j}-j}(M R)^{2^{j+1}-2} .
$$

Proof. - We imitate the well-known proof of Hua's Lemma, noting that for any positive integer $l$, as an easy exercise in estimates for the divisor function, one has that the number of integral solutions of the equation $m_{1}^{6}-m_{2}^{6}=l$ is $\mathrm{O}\left(l^{\varepsilon}\right)$.

Next, in view of (4.32), when $j=1$ we find by orthogonality that the integral on the left hand side of (4.34) is equal to the number $I_{1}$ of integral solutions of the equation

$$
h_{1}^{3}\left(m_{1}^{6}-n_{1}^{6}\right)=h_{2}^{3}\left(m_{2}^{6}-n_{2}^{6}\right)
$$

with

$$
1 \leqslant h_{i} \leqslant H, \quad M<n_{i}<m_{i} \leqslant M R \quad(i=1,2) .
$$

Given a fixed choice of $h_{2}, m_{2}, n_{2}$ satisfying (4.36), write $l=h_{2}^{3}\left(m_{2}^{6}-n_{2}^{6}\right)$. Then, for any solution $h_{1}, m_{1}, n_{1}$ of (4.35) counted by $I_{1}$, one has that $h_{1}$ and $m_{1}^{6}-n_{1}^{6}$ are each divisors of the positive integer $l$. On noting our opening discussion, elementary estimates for the divisor function therefore show that $I_{1} \ll P^{\varepsilon} H(M R)^{2}$, which confirms (4.34) when $j=1$.

By Cauchy's inequality, it follows from (4.32) that

$$
\int_{0}^{1}\left|E^{*}(\alpha)\right|^{4} \mathrm{~d} \alpha \ll(M R)^{2} \int_{0}^{1} E_{1}^{*}(\alpha)\left|E^{*}(\alpha)\right|^{2} \mathrm{~d} \alpha,
$$

where

$$
E_{1}^{*}(\alpha)=\sum_{M<m_{2}<m_{1} \leqslant M R}\left|\sum_{h \leqslant H} e\left(\alpha h^{3}\left(m_{1}^{6}-m_{2}^{6}\right)\right)\right|^{2} .
$$


On isolating the diagonal contribution, it follows by considering the underlying diophantine equations that

$$
\int_{0}^{1}\left|E^{*}(\alpha)\right|^{4} \mathrm{~d} \alpha \ll H(M R)^{4} \int_{0}^{1}\left|E^{*}(\alpha)\right|^{2} \mathrm{~d} \alpha+(M R)^{2} I_{2},
$$

where $I_{2}$ denotes the number of integral solutions of the equation

$$
h_{1}^{3}\left(m_{1}^{6}-n_{1}^{6}\right)-h_{2}^{3}\left(m_{2}^{6}-n_{2}^{6}\right)=\left(g_{1}^{3}-g_{2}^{3}\right)\left(m_{3}^{6}-n_{3}^{6}\right),
$$

with $\mathbf{g}, \mathbf{h}, \mathbf{m}, \mathbf{n}$ satisfying (4.36) and

$$
1 \leqslant g_{2}<g_{1} \leqslant H, \quad M<n_{3}<m_{3} \leqslant M R .
$$

Given a fixed choice of $h_{1}, h_{2}, m_{1}, m_{2}, n_{1}, n_{2}$ satisfying (4.36), write

$$
l=h_{1}^{3}\left(m_{1}^{6}-n_{1}^{6}\right)-h_{2}^{3}\left(m_{2}^{6}-n_{2}^{6}\right) .
$$

Then, for any solution $g_{1}, g_{2}, m_{3}, n_{3}$ of (4.38) counted by $I_{2}$, one has $l \neq 0$, and moreover the integers $g_{1}-g_{2}, g_{1}^{2}+g_{1} g_{2}+g_{2}^{2}$ and $m_{3}^{6}-n_{3}^{6}$ are each divisors of $l$. On recalling our opening discussion again, therefore, we find that $I_{2} \ll P^{\varepsilon} H^{2}(M R)^{4}$. The estimate (4.34) when $j=2$ is therefore confirmed on recalling (4.37), together with the estimate (4.34) in the case $j=1$. This completes the proof of the lemma.

LEMMA 4.6. - One has

$$
\int_{0}^{1}\left|F_{1}(\alpha)^{2} E^{*}\left(3 a^{2} \alpha\right)^{4}\right| \mathrm{d} \alpha \ll P^{\varepsilon} H^{5}(M R)^{11} .
$$

Proof. - Note first that by Cauchy's inequality, one has

$$
\left|F_{1}(\alpha)\right|^{2} \ll H(M R)^{2} \sum_{h \leqslant H}\left|\sum_{y \leqslant 2 A N} e\left(\alpha h y^{2}\right)\right|^{2} .
$$

Thus, on considering the underlying diophantine equations, one has the upper bound

$$
\int_{0}^{1}\left|F_{1}(\alpha)^{2} E^{*}\left(3 a^{2} \alpha\right)^{4}\right| \mathrm{d} \alpha \ll H(M R)^{2} J
$$

where $J$ denotes the number of integral solutions of the equation

$$
h\left(y_{1}^{2}-y_{2}^{2}\right)=3 a^{2}\left(h_{1}^{3}\left(m_{1}^{6}-n_{1}^{6}\right)+h_{2}^{3}\left(m_{2}^{6}-n_{2}^{6}\right)-h_{3}^{3}\left(m_{3}^{6}-n_{3}^{6}\right)-h_{4}^{3}\left(m_{4}^{6}-n_{4}^{6}\right)\right),
$$

with

$$
1 \leqslant h \leqslant H, \quad 1 \leqslant h_{i} \leqslant H, \quad M<n_{i}<m_{i} \leqslant M R \quad(1 \leqslant i \leqslant 4)
$$




$$
1 \leqslant y_{1} \leqslant 2 A N, \quad 1 \leqslant y_{2} \leqslant 2 A N .
$$

Let $J_{1}$ be the number of solutions of (4.41) counted by $J$ in which $y_{1}=y_{2}$, and let $J_{2}$ be the corresponding number of solutions with $y_{1} \neq y_{2}$. Then

$$
J=J_{1}+J_{2} .
$$

On considering the underlying diophantine equations, one has

$$
J_{1} \ll H N \int_{0}^{1}\left|E^{*}\left(3 a^{2} \alpha\right)\right|^{4} \mathrm{~d} \alpha,
$$

whence by making an obvious change of variable, it follows from Lemma 4.5 that

$$
J_{1} \ll N^{1+\varepsilon} H^{3}(M R)^{6} \ll P^{\varepsilon} H^{4}(M R)^{9} .
$$

Consider next a solution $h, \mathbf{y}, \mathbf{h}, \mathbf{m}, \mathbf{n}$ of Eq. (4.41) counted by $J_{2}$. Since now $y_{1} \neq y_{2}$, the integer on the right hand side of (4.41) must be non-zero. Fix any one of the $\mathrm{O}\left(H^{4}(M R)^{8}\right)$ possible choices of $\mathbf{h}, \mathbf{m}, \mathbf{n}$ with the latter property. Then $h, y_{1}-y_{2}$ and $y_{1}+y_{2}$ are each divisors of the fixed integer on the right hand side of (4.41), whence an elementary estimate for the divisor function shows that

$$
J_{2} \ll N^{\varepsilon} H^{4}(M R)^{8} .
$$

On combining (4.40) and (4.42)-(4.44), the conclusion (4.39) is immediate.

Before launching our first major offensive on the minor arcs, we record here the estimate

$$
\int_{0}^{1}\left|t\left(\alpha ; Q_{1}\right)\right|^{5} \mathrm{~d} \alpha \ll Q_{1}^{\frac{44}{17}-\delta},
$$

which follows from the proof of Theorem 1.2 of Wooley [22] with $\delta=10^{-4}$.

Lemma 4.7. - Suppose that $0<\theta \leqslant \frac{1}{6}$. Then

$$
\int_{\mathfrak{p}}\left|F_{1}(\alpha) \tilde{G}_{1}(A \alpha) t\left(A \alpha ; Q_{1}\right)^{2}\right| \mathrm{d} \alpha \ll N^{\frac{1}{2}+\varepsilon} M R^{2} H^{\frac{7}{5}} Q_{1}^{\frac{88}{85}-\frac{2}{5} \delta}\left(\operatorname{card} \mathcal{Z}_{3}(N)\right)^{\frac{1}{2}} .
$$

Proof. - Write

$$
\mathcal{J}_{2}=\int_{0}^{1}\left|F_{1}(\alpha)\right|^{1 / 5}\left|\tilde{G}_{1}(A \alpha) t\left(A \alpha ; Q_{1}\right)^{2}\right| \mathrm{d} \alpha
$$

and

$$
\mathcal{J}_{3}=\int_{0}^{1}\left|F_{1}(\alpha)\right|^{1 / 5}\left|E^{*}\left(3 a^{2} \alpha\right)\right|^{2 / 5}\left|\tilde{G}_{1}(A \alpha) t\left(A \alpha ; Q_{1}\right)^{2}\right| \mathrm{d} \alpha .
$$

Then by (4.30) and Lemma 4.4, one has for each $\alpha \in \mathfrak{p}$ the estimate

$$
\left|F_{1}(\alpha)\right| \ll N^{\varepsilon}(N H)^{1 / 2}|E(\alpha)|^{1 / 2},
$$


whence by (4.33) one finds that for $\alpha \in \mathfrak{p}$ one has

$$
\left|F_{1}(\alpha)\right|^{4 / 5} \ll N^{\varepsilon}(N H)^{2 / 5}\left((H M R)^{2 / 5}+\left|E^{*}\left(3 a^{2} \alpha\right)\right|^{2 / 5}\right) .
$$

Thus we obtain

$$
\int_{\mathfrak{p}}\left|F_{1}(\alpha) \tilde{G}_{1}(A \alpha) t\left(A \alpha ; Q_{1}\right)^{2}\right| \mathrm{d} \alpha \ll N^{\varepsilon}(N H)^{2 / 5}\left((H M R)^{2 / 5} \mathcal{J}_{2}+\mathcal{J}_{3}\right) .
$$

We first estimate $\mathcal{J}_{2}$, noting that by Hölder's inequality together with a change of variable, one has from (4.46) the bound

$$
\mathcal{J}_{2} \ll\left(\int_{0}^{1}\left|F_{1}(\alpha)\right|^{2} \mathrm{~d} \alpha\right)^{1 / 10}\left(\int_{0}^{1}\left|\tilde{G}_{1}(\alpha)\right|^{2} \mathrm{~d} \alpha\right)^{1 / 2}\left(\int_{0}^{1}\left|t\left(\alpha ; Q_{1}\right)\right|^{5} \mathrm{~d} \alpha\right)^{2 / 5} .
$$

It therefore follows from Lemma 4.3 and (4.45) that

$$
\mathcal{J}_{2} \ll N^{\varepsilon}(N M H R)^{1 / 10}\left(M R H \operatorname{card} \mathcal{Z}_{3}(N)\right)^{1 / 2}\left(Q_{1}^{\frac{44}{17}-\delta}\right)^{2 / 5} .
$$

Meanwhile, on applying Hölder's inequality to (4.47), we find that

$$
\mathcal{J}_{3} \ll\left(\int_{0}^{1}\left|F_{1}(\alpha) E^{*}\left(3 a^{2} \alpha\right)^{2}\right|^{2} \mathrm{~d} \alpha\right)^{1 / 10}\left(\int_{0}^{1}\left|\tilde{G}_{1}(\alpha)\right|^{2} \mathrm{~d} \alpha\right)^{1 / 2}\left(\int_{0}^{1}\left|t\left(\alpha ; Q_{1}\right)\right|^{5} \mathrm{~d} \alpha\right)^{2 / 5} .
$$

By making use of Lemmata 4.3 and 4.6, therefore, we deduce from (4.45) that

$$
\mathcal{J}_{3} \ll N^{\varepsilon}\left(H^{5}(M R)^{11}\right)^{1 / 10}\left(M R H \operatorname{card} \mathcal{Z}_{3}(N)\right)^{1 / 2}\left(Q_{1}^{\frac{44}{17}-\delta}\right)^{2 / 5} .
$$

On recalling (4.48), we conclude from (4.49) and (4.50) that

$$
\int_{\mathfrak{p}}\left|F_{1}(\alpha) \tilde{G}_{1}(A \alpha) t\left(A \alpha ; Q_{1}\right)^{2}\right| \mathrm{d} \alpha \ll N^{\frac{1}{2}+\varepsilon} M R H^{7 / 5} Q_{1}^{\frac{88}{85}-\frac{2}{5} \delta}\left(\operatorname{card} \mathcal{Z}_{3}(N)\right)^{1 / 2}(1+\omega),
$$

where

$$
\omega \ll(M R)^{3 / 5} N^{-1 / 10} .
$$

Consequently our hypothesis that $\theta \leqslant \frac{1}{6}$ ensures that $\omega \ll R^{3 / 5}$, and the conclusion of the lemma follows immediately.

A further mean value estimate is necessary before dispatching the contribution of the major $\operatorname{arcs} \mathfrak{P}$.

Lemma 4.8. - One has

$$
\int_{\mathfrak{P}} D(\alpha)\left|t\left(A \alpha ; Q_{1}\right)\right|^{2} \mathrm{~d} \alpha \ll N^{3+\varepsilon} H Q_{1}^{-2}
$$


Proof. - It follows from Lemma 4.4 that

$$
\int_{\mathfrak{P}} D(\alpha)\left|t\left(A \alpha ; Q_{1}\right)\right|^{2} \mathrm{~d} \alpha \ll N^{2+\varepsilon} H \int_{\mathfrak{P}} D^{*}(\alpha) \Psi(\alpha) \mathrm{d} \alpha,
$$

where for $\alpha \in \mathfrak{P}(q, a) \subset \mathfrak{P}$ one has

$$
D^{*}(\alpha)=q^{-1}\left(1+Q_{1}^{3}|\alpha-a / q|\right)^{-1}
$$

and where we define

$$
\Psi(\alpha)=\left|t\left(A \alpha ; Q_{1}\right)\right|^{2}=\sum_{|h| \leqslant 2 A Q_{1}^{3}} \psi_{h} e(\alpha h) .
$$

Here $\psi_{h}$ denotes the number of solutions of the equation $h=A\left(x_{1}^{3}-x_{2}^{3}\right)$ with $x_{1}, x_{2} \in$ $\mathcal{A}\left(Q_{1}, R\right)$. As an immediate consequence of Lemma 2 of Brüdern [1], we have

$$
\int_{\mathfrak{P}} D^{*}(\alpha)\left|t\left(A \alpha ; Q_{1}\right)\right|^{2} \mathrm{~d} \alpha \ll N^{\varepsilon} Q_{1}^{-3}\left(P \psi_{0}+\sum_{h \neq 0}\left|\psi_{h}\right|\right) \ll N^{\varepsilon} Q_{1}^{-3}\left(P Q_{1}+Q_{1}^{2}\right),
$$

and the conclusion of the lemma follows immediately.

LEMmA 4.9. - Suppose that $0<\theta \leqslant \frac{1}{6}$. Then

$$
\begin{aligned}
& \int_{0}^{1}\left|F_{1}(\alpha) \tilde{G}_{1}(A \alpha) t\left(A \alpha ; Q_{1}\right)^{2}\right| \mathrm{d} \alpha \\
& \quad \ll N^{3+\varepsilon} H^{2} M^{2} R^{2} Q_{1}^{-2}+N^{\frac{1}{2}+\varepsilon} M R^{2} H^{\frac{7}{5}} Q_{1}^{\frac{88}{85}-\frac{2}{5} \delta}\left(\operatorname{card} \mathcal{Z}_{3}(N)\right)^{1 / 2} .
\end{aligned}
$$

Proof. - Note that $[0,1]$ is the disjoint union of $\mathfrak{P}$ and $\mathfrak{p}$. Therefore, if one has

$$
\int_{\mathfrak{p}}\left|F_{1}(\alpha) \tilde{G}_{1}(A \alpha) t\left(A \alpha ; Q_{1}\right)^{2}\right| \mathrm{d} \alpha \geqslant \int_{\mathfrak{P}}\left|F_{1}(\alpha) \tilde{G}_{1}(A \alpha) t\left(A \alpha ; Q_{1}\right)^{2}\right| \mathrm{d} \alpha
$$

then

$$
\int_{0}^{1}\left|F_{1}(\alpha) \tilde{G}_{1}(A \alpha) t\left(A \alpha ; Q_{1}\right)^{2}\right| \mathrm{d} \alpha \leqslant 2 \int_{\mathfrak{p}}\left|F_{1}(\alpha) \tilde{G}_{1}(A \alpha) t\left(A \alpha ; Q_{1}\right)^{2}\right| \mathrm{d} \alpha,
$$

and the conclusion of the lemma is immediate from Lemma 4.7. In the contrary case, the same argument, followed by an application of Schwarz's inequality, yields

$$
\begin{aligned}
& \int_{0}^{1}\left|F_{1}(\alpha) \tilde{G}_{1}(A \alpha) t\left(A \alpha ; Q_{1}\right)^{2}\right| \mathrm{d} \alpha \\
& \quad \leqslant 2 \int_{\mathfrak{P}}\left|F_{1}(\alpha) \tilde{G}_{1}(A \alpha) t\left(A \alpha ; Q_{1}\right)^{2}\right| \mathrm{d} \alpha \leqslant 2\left(K_{1} K_{2}\right)^{1 / 2},
\end{aligned}
$$


where

$$
K_{1}=\int_{\mathfrak{P}}\left|F_{1}(\alpha) t\left(A \alpha ; Q_{1}\right)\right|^{2} \mathrm{~d} \alpha
$$

and

$$
K_{2}=\int_{0}^{1}\left|\tilde{G}_{1}(A \alpha) t\left(A \alpha, Q_{1}\right)\right|^{2} \mathrm{~d} \alpha .
$$

Observe first that on considering the underlying diophantine equations, one may apply the argument leading from (4.23) to the conclusion of Lemma 4.2 in order to establish that

$$
K_{2} \leqslant \int_{0}^{1}\left|F_{1}(\alpha) \tilde{G}_{1}(A \alpha) t\left(A \alpha ; Q_{1}\right)^{2}\right| \mathrm{d} \alpha .
$$

Thus it follows from (4.51)-(4.53) that

$$
\int_{0}^{1}\left|F_{1}(\alpha) \tilde{G}_{1}(A \alpha) t\left(A \alpha ; Q_{1}\right)^{2}\right| \mathrm{d} \alpha \ll \int_{\mathfrak{P}}\left|F_{1}(\alpha) t\left(A \alpha ; Q_{1}\right)\right|^{2} \mathrm{~d} \alpha .
$$

Next, on making use of (4.30) together with a trivial estimate for $E(\alpha)$, we obtain

$$
\int_{\mathfrak{P}}\left|F_{1}(\alpha) t\left(A \alpha ; Q_{1}\right)\right|^{2} \mathrm{~d} \alpha \leqslant H(M R)^{2} \int_{\mathfrak{P}} D(\alpha)\left|t\left(A \alpha ; Q_{1}\right)\right|^{2} \mathrm{~d} \alpha,
$$

whence by Lemma 4.8 we may conclude that

$$
\int_{\mathfrak{P}}\left|F_{1}(\alpha) t\left(A \alpha ; Q_{1}\right)\right|^{2} \mathrm{~d} \alpha \ll N^{3+\varepsilon} H^{2}(M R)^{2} Q_{1}^{-2} .
$$

The proof of the lemma is completed by substituting (4.55) into (4.54).

Having now vanquished the difficult aspects of estimating the central auxiliary mean value, we set up camp by collecting together our conclusions in the form of the following lemma.

Lemma 4.10. - Suppose that card $\mathcal{Z}_{3}(N)>N^{78 / 85}$. Then, with $\tau=10^{-6}$, one has

$$
\int_{0}^{1}\left|K_{3}(\alpha)^{2} t(\alpha ; P)^{4}\right| \mathrm{d} \alpha \ll N^{\frac{5216}{1865}-\tau}\left(\operatorname{card} \mathcal{Z}_{3}(N)\right)^{\frac{169}{373}} .
$$

Proof. - We choose $\theta$ by taking $M=P^{\theta}$ and solving the equation

$$
M=P^{\frac{743}{1865}-2 \tau}\left(\operatorname{card} \mathcal{Z}_{3}(N)\right)^{-\frac{102}{373}} .
$$

On making use of our hypothesis that $\operatorname{card} \mathcal{Z}_{3}(N)>N^{78 / 85}$, we find that $P^{1 / 9}<M<P^{1 / 6}$, and hence that the estimate of Lemma 4.9 holds. Moreover, our hypothesis on the cardinality of $\mathcal{Z}_{3}(N)$ also ensures that the estimate of Lemma 4.9 may be simplified to 


$$
\begin{aligned}
& \int_{0}^{1}\left|F_{1}(\alpha) \tilde{G}_{1}(A \alpha) t\left(A \alpha ; Q_{1}\right)^{2}\right| \mathrm{d} \alpha \\
& \quad \ll N^{\frac{1}{2}+\varepsilon} M R^{2} H^{\frac{7}{5}} Q_{1}^{\frac{88}{85}-\frac{2}{5} \delta}\left(\operatorname{card} \mathcal{Z}_{3}(N)\right)^{1 / 2} .
\end{aligned}
$$

On recalling the estimate

$$
\int_{0}^{1}\left|t\left(\alpha ; Q_{1}\right)\right|^{6} \mathrm{~d} \alpha \ll Q_{1}^{\frac{13}{4}-4 \delta}
$$

from Theorem 1.2 of Wooley [22], we deduce from Lemma 4.2 and (4.56) that

$$
\int_{0}^{1}\left|G_{1}(\alpha) t\left(\alpha ; Q_{1}\right)^{4}\right| \mathrm{d} \alpha \ll N^{\frac{1}{4}+\varepsilon} M^{1 / 2} R H^{7 / 10} Q_{1}^{\frac{1457}{680}-2 \delta}\left(\operatorname{card} \mathcal{Z}_{3}(N)\right)^{1 / 4}
$$

On inserting the latter estimate into Lemma 4.1, and taking account of our choice of $M$, a modicum of computation will yield the conclusion of the lemma whenever $\eta$ is sufficiently small.

Having achieved the modest victory recorded above, we manoeuvre our forces to outflank the obstacles on the major arcs by means of a pruning argument. Given the work of the previous section, this turns out to be routine. We start once again with the lower bound (2.28), and investigate the contribution to the left hand side of (2.28) arising from the set $\mathfrak{n} \cap \mathfrak{M}$. Recalling the notation of §3, we apply Lemmata 7.2 and 8.5 of Vaughan and Wooley [18] to conclude that for $X \leqslant P^{3 / 4}$ one has

$$
\sup _{\alpha \in \mathfrak{K}(X)}|t(\alpha)| \ll P X^{-1 / 8}
$$

Then it follows from Lemma 3.3 that for $X \leqslant P^{3 / 4}$ one has

$$
\begin{aligned}
& \int_{\mathfrak{K}(X)}\left|\mathcal{F}_{1}(\alpha) t(\alpha)^{2} K_{3}(\alpha)\right| \mathrm{d} \alpha \\
& \ll P^{2} X^{-1 / 4} K_{3}(0) \int_{\mathfrak{M}(2 X)}\left|\mathcal{F}_{1}(\alpha)\right| \mathrm{d} \alpha \\
& \quad \ll Q^{3} Y X^{-1 / 5}(\log Y)^{-1} \operatorname{card} \mathcal{Z}_{3}(N) .
\end{aligned}
$$

On summing over the contributions of the sets $\mathfrak{K}\left(2^{l} L\right)$ with $l \geqslant 0$ and $2^{l} L \leqslant P^{3 / 4}$, we obtain the upper bound

$$
\int_{\mathfrak{n} \cap \mathfrak{M}}\left|\mathcal{F}_{1}(\alpha) t(\alpha)^{2} K_{3}(\alpha)\right| \mathrm{d} \alpha \ll Q^{3} Y L^{-1 / 5}(\log Y)^{-1} \operatorname{card} \mathcal{Z}_{3}(N) .
$$

On recalling (2.28), we may conclude thus far that 


$$
\int_{\mathfrak{m}}\left|\mathcal{F}(\alpha) t(\alpha)^{2} K_{3}(\alpha)\right| \mathrm{d} \alpha+\int_{\mathfrak{M}}\left|\left(\mathcal{F}(\alpha)-\mathcal{F}_{1}(\alpha)\right) t(\alpha)^{2} K_{3}(\alpha)\right| \mathrm{d} \alpha
$$

$$
\gg Q^{3} Y(\log Y)^{-1} \operatorname{card} \mathcal{Z}_{3}(N) .
$$

On applying Schwarz's inequality next to (4.57), and making use of Lemmata 3.2 and 4.10, we find that whenever card $\mathcal{Z}_{3}(N)>N^{78 / 85}$, one has the estimate

$$
\begin{aligned}
Q^{3} Y(\log Y)^{-1} \operatorname{card} \mathcal{Z}_{3}(N) \ll & \left(\int_{\mathfrak{m}}|\mathcal{F}(\alpha)|^{2} \mathrm{~d} \alpha+\int_{\mathfrak{M}}\left|\mathcal{F}(\alpha)-\mathcal{F}_{1}(\alpha)\right|^{2} \mathrm{~d} \alpha\right)^{1 / 2} \\
& \times\left(\int_{0}^{1}\left|K_{3}(\alpha)^{2} t(\alpha ; P)^{4}\right| \mathrm{d} \alpha\right)^{1 / 2} \\
\ll & \left(Y^{2} Q^{6} P^{-19 / 14}\right)^{1 / 2}\left(N^{\frac{5216}{1865}-\tau}\right)^{1 / 2}\left(\operatorname{card} \mathcal{Z}_{3}(N)\right)^{\frac{169}{746}} .
\end{aligned}
$$

It follows that

$$
\operatorname{card} \mathcal{Z}_{3}(N) \ll N^{1-\frac{2801}{40390}},
$$

and the conclusion of Theorem 1.2 follows from a trivial computation.

\section{REFERENCES}

[1] BRÜDERn J., A problem in additive number theory, Math. Proc. Cambridge Philos. Soc. 103 (1988) $27-33$.

[2] BRÜDERn J., On Waring's problem for cubes, Math. Proc. Cambridge Philos. Soc. 109 (1991) 229256.

[3] BRÜDERn J., KAWADA K., WoOley T.D., Additive representation in thin sequences, II: the binary Goldbach problem, Mathematika, to appear.

[4] BRÜDERN J., KAWADA K., WOOLEY T.D., Additive representation in thin sequences, III: asymptotic formulae, Acta Arith., to appear.

[5] BRÜDERN J., KAWADA K., WOOLEY T.D., Additive representation in thin sequences, IV: lower bound methods, Quart. J. Math. Oxford, to appear.

[6] BRÜDERn J., KAWADA K., WOOley T.D., Additive representation in thin sequences, V: mixed problems of Waring's type, Math. Scand., to appear.

[7] BRÜDERN J., WatT N., On Waring's problem for four cubes, Duke Math. J. 77 (1995) 583-606.

[8] DAVENPORT H., On Waring's problem for cubes, Acta Math. 71 (1939) 123-143.

[9] Davenport H., Analytic Methods for Diophantine Equations and Diophantine Inequalities, Ann Arbor Publishers, Ann Arbor, 1962.

[10] Estermann T., Einige Sätze über quadratfreie Zahlen, Math. Ann. 105 (1931) 653-662.

[11] Hardy G.H., Littlewood J.E., Some problems of "Partitio Numerorum". I, A new solution of Waring's problem, Göttinger Nachrichten (1920) 33-54.

[12] KaWADA K., On the sum of four cubes, Mathematika 43 (1996) 323-348.

[13] LiNNIK J.V., On the representation of large numbers as sums of seven cubes, Mat. Sbornik 12 (1943) 218-224.

[14] Vaughan R.C., On Waring's problem for cubes, J. Reine Angew. Math. 365 (1986) 122-170.

[15] Vaughan R.C., A new iterative method in Waring's problem, Acta Math. 162 (1989) 1-71.

[16] Vaughan R.C., On Waring's problem for cubes II, J. London Math. Soc. 39 (2) (1989) 205-218.

$4^{\mathrm{e}}$ SÉRIE - TOME $34-2001-\mathrm{N}^{\circ} 4$ 
[17] Vaughan R.C., The Hardy-Littlewood Method, 2nd. ed., Cambridge University Press, Cambridge, 1997.

[18] Vaughan R.C., Wooley T.D., On Waring's problem: some refinements, Proc. London Math. Soc. 63 (3) (1991) 35-68.

[19] Watson G.L., A proof of the seven cube theorem, J. London Math. Soc. 26 (1951) 153-156.

[20] Wooley T.D., On simultaneous additive equations, II, J. Reine Angew. Math. 419 (1991) 141-198.

[21] Wooley T.D., Large improvements in Waring's problem, Ann. of Math. 135 (2) (1992) 131-164.

[22] WoOLEY T.D., Breaking classical convexity in Waring's problem: sums of cubes and quasi-diagonal behaviour, Invent. Math. 122 (1995) 421-451.

Jörg BRÜDERN

Mathematisches Institut A,

Universität Stuttgart,

Pfaffenwaldring 57,

D-70511 Stuttgart, Germany

Koichi KAWADA

Department of Mathematics,

Faculty of Education,

Iwate University,

Morioka 020-8550, Japan

Trevor D. Wooley

Department of Mathematics,

University of Michigan,

East Hall, 525 East University Avenue,

Ann Arbor, MI 48109-1109, USA 